\title{
Morphological evolution of dwarf galaxies in the Local Group
}

\author{
S. Pasetto, C. Chiosi, and G. Carraro
}

\author{
Department of Astronomy, University of Padova, Vicolo dell'Osservatorio 2, 35122 Padova, Italy \\ e-mail: chiosi@pd.astro.it; carraro@pd.astro.it
}

Received 2 April 2002 / Accepted 16 April 2003

\begin{abstract}
The dwarf galaxies of the Local Group can be separated in three morphological groups: irregular, elliptical and spheroidal. As in the large galaxy clusters, there seems to be a morphology-position relationship: irregular galaxies are preferentially found in the outskirts (low density regions) of the Local Group, whereas dwarf ellipticals and spheroidals are more frequent in the central, high density regions. To cast light on the nature and origin of dwarf galaxies in the Local Group, Mayer et al. (2001a) have suggested that a dwarf irregular galaxy tidally interacting with a galaxy of much larger mass may be reshaped into a dwarf spheroidal or elliptical object. In this paper we check by means of $N$-body Tree-SPH simulations whether this is possible for a selected sample of galaxies of the Local Group. Using the best data available in literature to fix the dynamical and kinematical status of a few dwarf galaxies in the Local Group, we follow the evolution of an ideal satellite, which supposedly started as an irregular object during its orbital motion around the Milky Way. We find that the tidal interactions with the Milky Way remove a large fraction of the mass of the dwarf irregular and gradually reshape it into a spherical object.
\end{abstract}

Key words. galaxies: Local Group - galaxies: dwarf - galaxies: evolution - galaxies: interactions methods: $N$-body simulations

\section{Introduction}

Dwarf Galaxies (dGs) are the most common objects in the Universe (Marzke \& Da Costa 1997) and those in the Local Group (LG) allow us to study a unique sample of this kind of galaxies (Binney 2000). According to Mateo (1998), van den Bergh (1999) and Grebel (1999), the LG contains about $40 \mathrm{dGs}$ with absolute magnitude $M_{B} \in[-18,-9]$ that are morphologically grouped as irregular (dIrrs), elliptical (dEs), and spheroidal (dSphs).

The absolute magnitude of dEs is $M_{B}>-17$ and their typical surface brightness is $\mu_{B} \leq 21 \mathrm{mag} \mathrm{arcsec}{ }^{-2}$. The compact dEs (Kormendy 1977, 1987; Kormendy \& Djorgovski 1989) - the only example of which in the LG is M 32 - show radial surface brightness profiles that fit the de Vaucouleurs $R^{1 / 4}$ law like in giant ellipticals. On the contrary, the diffuse dEs have surface brightness profiles that can be approximated by an exponential law (Mateo 1998). Furthermore, studies of the apparent ellipticity show that the degree of flattening is not very large. The dEs contain mainly old stars ( $>10 \mathrm{Gyr})$. Some of them, however, show also evidence of recent star formation (1-10 Gyr).

The dSphs are among the faintest known galaxies in the sky with $M_{B}>-14$ and $\mu_{B} \geq 20 \mathrm{mag} \operatorname{arcsec}^{-2}$. They contain old and/or intermediate-age stellar populations.

Send offprint requests to: $\mathrm{S}$. Pasetto, e-mail: pasetto@pd.astro.it
Both dEs and dSphs are kinematically supported by their velocities dispersion: typically the ratio of rotational velocity to the velocity dispersion $v / \sigma<0.5$ (Mateo 1998; Ferguson $\&$ Binggeli 1994). Finally, their gas abundance is very low as indicated by the ratio of the $\mathrm{HI}$ mass, $M_{\mathrm{HI}}$, to the total baryonic mass (gas+stars), $M_{\mathrm{BM}}, M_{\mathrm{HI}} / M_{\mathrm{BM}}<0.1$.

The dIrrs are the objects with the most irregular shape. They have low density and low surface brightness $\mu_{B} \cong 23$, and contain regions of ongoing star formation full of $\mathrm{OB}$ associations and HII regions that are superposed on an underlying diffuse structure of old stars with an exponential surface brightness profile. In general, they are rich of HI (Hoffman et al. 1996) in rotation with $v / \sigma>1$ and concentrated in clouds of 100-300 pc diameter. The fractional mass abundance of $\mathrm{HI}$ with respect to $M_{\mathrm{BM}}$ is $M_{\mathrm{HI}} / M_{\mathrm{BM}} \simeq 0.5$ to 0.7 , to which the contribution from molecular HII, gas of other nature, and dust has to be added.

A small number of galaxies exist whose properties are intermediate between dIrrs and dSphs-dEs. They could suggest evolution from dIrrs to dSphs (Grebel 1999).

All dwarf galaxies appear to be Dark Matter (DM) dominated and show evidence of an anti-correlation between their total absolute magnitude and mass-to-light ratio $M / L$, e.g. the DSph Draco and Ursa Minor with $M_{B}>-10$ have $M / L>25$ whereas GR8 and SagDIG with $M_{B}>-12$ have $M / L \cong 30$.

Dwarf galaxies in the LG seem to obey a morphologylocal density relation (Grebel 1999): in the vicinity of the Milky Way (MW) and M 31, let say within 300-400 kpc of the 
dominant galaxy, there are mainly dSphs and dEs that most likely are satellites of the giant spirals and orbit inside the deep potential well of the DM halo of the dominant object (Peebles et al. 1989). In contrast, dIrrs are preferentially found in the outskirts of the LG (van den Bergh 1996).

Looking at the few transition objects between dSphs-dEs and dIrrs suggesting a genetic relationship between these systems, a mechanism must be found by which cold gas is removed from dIrrs thus reducing their angular moment, kinematically heating their pre-existing stellar populations, and reshaping dIrrs into dSphs-dEs.

Various mechanisms have been proposed. Gas could be expelled by galaxies with low spin velocity $\left(V_{\mathrm{c}} \leq 100 \mathrm{~km} \mathrm{~s}^{-1}\right)$, thanks to the energy feedback by supernova explosions. This could explain the relationship between luminosity and surface brightness of the dIrrs and dSphs. However, as argued by Mac Low \& Ferrara (1999), this mechanism would require a DM halo substantially less massive than currently attributed to dGs (Mateo 1998). Furthermore, gas loss would not account for the observational difference in angular momentum between dIrrs and dSphs.

Alternatively, ram pressure exerted by a gaseous halo surrounding the disk of the main galaxy could undress the dGs while crossing the halo because of their weak gravitational binding energy. However, recent studies by Murali (2000) have pointed out that this mechanism is not likely to be efficient at least in the case of our own galaxy owing to the low density of the Galactic Halo.

Finally, galaxy harassment has been invoked to justify the loss of mass and angular moment (Moore et al. 1996b, 1998, 1999a). However it has been argued that galaxy harassment could be effective in high density clusters like Coma and not in the LG (Mayer et al. 2001a) where the encounters among $\mathrm{dGs}$, satellites of a dominant object, are so rare that they never perturb their mutual orbits.

Therefore we are left with the tidal interaction between a dominant galaxy and the small satellites as the agent altering the dynamical structure of these latter (Mayer et al. 2001a). In this context, Weinberg $(1994 a-c)$ and Gnedin et al. (1999a,b) have argued that strong dynamical heating of the satellites by tidal effects may occur in the case of high eccentricity elliptical orbits. In addition to this, the above mechanism by slowly changing the orbital motion of the satellite could lead the large galaxy to engulf the small one, e.g. Sagittarius in the MW. Along this vein, the Galactic Halo could result from merging smaller sub-units up to a mass percentage as large as 50\% (Mateo 1998). This is indeed predicted by hierarchical clustering scenario according to which former small irregular structures are organized in large more regular bodies. Morphological properties of globular clusters in nearby galaxies seem also to support this view. While the globular clusters of the MW and M31 are nearly spherical, those of LMC or SMC are significantly elongated (the LMC clusters have modal ellipticity of 0.85 ). It is probable that different ellipticities mirror systematic age differences (Han \& Ryden 1994): under the action of dynamical interactions globular clusters become more and more spherical at increasing age. Indeed the majority of LMC globular clusters are younger than about 3-4 Gyr (Da Costa 1990) whereas those of the MW are older than $10 \mathrm{Gyr}$.

In this study, by means of dynamical $N$-body Tree-SPH simulations, we try to investigate whether a dIrr can evolve into a dSph as a result of dynamical interactions with a galaxy of much larger mass. With respect to the study of Mayer et al. (2001a), the analysis is made for a selected sample of dGs of the LG. Using literature data for the proper motions and radial velocities of dGs of the $\mathrm{LG}$, we set the orbital parameters of the dGs interacting with the MW. A suitable description for the gravitational potential due to the main components of the MW (Halo, Bulge and Disk) is adopted to calculate the gravitational interaction with the dGs. Finally, the dynamical and geometrical structures of the $\mathrm{dG}$ are followed for a period of time comparable to that of several revolutions about the MW.

The plan of the paper is as follows. In Sect. 2 we set-up the initial conditions of the motion of the satellite dIrr galaxy. In Sect. 3 we construct an initial model for dIrrs. In Sect. 4 we check the dynamical and morphological stability of a dIrr galaxy evolved in isolation for a period of time corresponding 3 to 4 passages at the galactic peri-center (about $6 \mathrm{Gyr}$ ) of the orbital motion about the MW. In Sect. 5, the dIrr model galaxy is put in orbit around the MW. In Sect. 6 we present the effects of the tidal interactions on the morphology of the $\mathrm{dG}$. We find that on a rather short time scale, a dIrr can re-shaped in a dSph of smaller mass. In Sect. 7 we examine whether the newly borne $\mathrm{dSph}$ is a stable configuration able to survive for long periods of time. In Sect. 8 we analyze whether the star formation history of the satellite galaxy may show traces (bursts of activity) of the close encounters with the MW. Finally, in Sect. 9 we present some concluding remarks.

\section{Initial conditions for the orbital motion}

In this section we derive the initial conditions for the orbital motion of a dG about a galaxy of larger mass (the MW in our case). The task is hampered by the very small number of galaxies for which good determinations of the proper motions from direct observations are currently available (Irwin 1998). The original sample of more than 40 galaxies belonging to the LG drastically reduce to 5 , all of them satellites of the MW. The orbital parameters for this small group of objects are listed in Table 1.

However, in addition to proper motions, the radial speed and parallax are required to derive the galacto-centric velocity, position and distance of the dG. To this aim we follow the method used by Johnson \& Soderblom (1987), however referred to the equinox J2000 (see also Green 1985), to derive the heliocentric speed of the $\mathrm{dG}$.

First, the equatorial coordinates $(\alpha, \delta)$ listed by Mateo (1998) for the galaxies in the sample of Table 1 are transformed into the Galactic Coordinate System $(b, l)$ by means of the following relationship

$\left[\begin{array}{c}\cos b \cos l \\ \cos b \sin l \\ \sin b\end{array}\right]=\mathcal{T}\left[\begin{array}{c}\cos \delta \cos \alpha \\ \cos \delta \sin \alpha \\ \sin \delta\end{array}\right]$ 
Table 1. Summary table of the basic parameters for the orbital motions of a selected sample of dGs of the LG. Column 1 lists the galaxy. Columns 2 and 3 are the galactic coordinates. The conversion from $\alpha$ and $\delta$ to $l_{\mathrm{J} 2000}$ and $b_{\mathrm{J} 2000}$ is as in Green (1985). In the case of LMC-SMC we refer to Wilkinson \& Evans (1999). Distances and associated dispersions, $D_{\text {hel }}$ and $\sigma_{D_{\text {hel }}}$ respectively, (Cols. 4 and 5), are referred to the Sun (Mateo 1998; Mighell \& Bruke 1999). The helio-centric velocities and their dispersion, (Cols. 6 and 7) $V_{R_{\text {hel }}}$ and $\sigma_{V_{R, \text { hel }}}$ respectively, are derived from optical and radio data (Mateo 1998). Finally, $\mu_{\alpha}, \sigma_{\mu, \alpha}, \mu_{\delta}$ and $\sigma_{\mu, \delta}$, (Cols. 8 through 11) are the proper motions and their dispersions. See also Binney \& Tremaine (1994) and Irwin (1998).

\begin{tabular}{|c|c|c|c|c|c|c|c|c|c|c|}
\hline Galaxy & $l_{\mathrm{J} 2000}$ & $b_{\mathrm{J} 2000}$ & $D_{\text {hel }}$ & $\sigma_{D_{\text {hel }}}$ & $V_{R_{\text {hel }}}$ & $\sigma_{V_{R, \text { hel }}}$ & $\mu_{\alpha}$ & $\sigma_{\mu, \alpha}$ & $\mu_{\delta}$ & $\sigma_{\mu, \delta}$ \\
\hline & \multicolumn{2}{|c|}{ degrees } & \multicolumn{2}{|c|}{$\mathrm{kpc}$} & \multicolumn{2}{|c|}{$\mathrm{km} \mathrm{s}^{-1}$} & \multicolumn{4}{|c|}{${\text { mas } \mathrm{yr}^{-1}}^{-1}$} \\
\hline Sculptor & 287.5 & -83.2 & 79.0 & 4 & 108 & 3 & 0.73 & 0.22 & -0.07 & 0.25 \\
\hline Ursa Minor & 105.0 & 44.8 & 69.4 & 4 & -248 & 2 & 0.06 & 0.08 & 0.07 & 0.10 \\
\hline Draco & 86.4 & 34.7 & 82.0 & 6 & -293 & 2 & 0.60 & 0.40 & 1.10 & 0.50 \\
\hline Sagittarius & 5.6 & -14.1 & 24.0 & 2 & 140 & 5 & -2.65 & 0.08 & -0.88 & 0.08 \\
\hline LMC/SMC & 282.0 & -34.0 & 49.0 & 2 & 274 & 3 & 1.61 & 0.19 & -0.06 & 0.25 \\
\hline
\end{tabular}

where the matrix $\mathcal{T}$ is the product of the three rotation matrices $\left(\mathcal{T}=\mathcal{T}_{1} \mathcal{T}_{2} \mathcal{T}_{3}\right)$

The matrix $\mathcal{T}_{1}$ corresponds to a rotation around the position angle of the North Celestial Pole with respect to the semi-circle passing through the North Galactic Pole and the zero Galactic longitude $\theta_{0}=122.7^{\circ}$

$\mathcal{T}_{1}=\left[\begin{array}{ccc}+\cos \theta_{0} & +\sin \theta_{0} & 0 \\ +\sin \theta_{0} & -\cos \theta_{0} & 0 \\ 0 & 0 & +1\end{array}\right]$.

The matrices $\mathcal{T}_{2}$ and $\mathcal{T}_{3}$ represent the rotation around the two equatorial position angles of the North Galactic Pole $\delta_{\mathrm{NGP}}=27^{\circ} 27^{\prime}$

$\mathcal{T}_{2}=\left[\begin{array}{ccc}+\sin \delta_{\mathrm{NGP}} & 0 & +\cos \delta_{\mathrm{NGP}} \\ 0 & -1 & 0 \\ +\cos \delta_{\mathrm{NGP}} & 0 & +\sin \delta_{\mathrm{NGP}}\end{array}\right]$

and $\alpha_{\mathrm{NGP}}=192^{\circ} 49^{\prime} 30^{\prime \prime}$, respectively,

$\mathcal{T}_{3}=\left[\begin{array}{ccc}+\cos \alpha_{\mathrm{NGP}} & +\sin \alpha_{\mathrm{NGP}} & 0 \\ +\sin \alpha_{\mathrm{NGP}} & -\cos \alpha_{\mathrm{NGP}} & 0 \\ 0 & 0 & +1\end{array}\right]$.

Adopting the coordinate matrix

$\mathcal{A}=\left[\begin{array}{ccc}+\cos \alpha \cos \delta & -\sin \alpha & -\cos \alpha \sin \delta \\ +\sin \alpha \cos \delta & +\cos \alpha & -\sin \alpha \sin \delta \\ +\sin \delta & 0 & +\cos \delta\end{array}\right]$

the three components of the velocity vector are given by

$\left[\begin{array}{c}U \\ V \\ W\end{array}\right]=\mathcal{T} A\left[\begin{array}{c}V_{r} \\ \frac{k \mu_{\alpha}}{\varpi} \\ \frac{k \mu_{\delta}}{\sigma}\end{array}\right]$

where $\varpi$ is the parallax in arcsec, and $k=4.74$ is a conversion factor between the units for the velocities $(U, V, W)$ we have chosen $\left(\mathrm{km} \mathrm{s}^{-1}\right)$ and the proper motions $\left(\mu_{\alpha}, \mu_{\delta}\right)$ in $\operatorname{arcsec} \mathrm{yr}^{-1}$. It is worth noting here that a right-handed coordinate system for $U, V$ and $W$ is adopted, so that the velocity components are positive in directions toward the Galactic center, the Galactic rotation, and the North Galactic Pole.

These values for the three components of the velocity vector are then corrected for the solar motion relative to the Local
Table 2. Components of the Galactocentric velocity vector $\left(V_{x}, V_{y}, V_{z}\right)$, where $V_{x}$ points in the direction of the sun, $V_{y}$ in the direction of the rotation of the galaxy and $V_{z}$ to form a left-handed system. The velocities refer to the motion of the galaxy barycentre. Thanks to the lucky circumstance that LMC and SMC move nearly parallel to each other (Kroupa \& Bastian 1997), the complex LMC+SMC is considered as a single object moving along the trajectory of the LMC but located at the barycentre of the system. The velocities are in $\mathrm{km} \mathrm{s}^{-1}$.

\begin{tabular}{lrrr}
\hline \hline Galaxy & $V_{x}$ & $V_{y}$ & $V_{z}$ \\
\hline Sculptor & -218.6 & +46.1 & -243.4 \\
Ursa Minor & +27.9 & +74.1 & -227.8 \\
Draco & +422.2 & +74.4 & -195.9 \\
Sagittarius & +204.7 & +35.6 & +281.3 \\
LMC/SMC & +29 & -63.7 & -206.5 \\
\hline
\end{tabular}

Standard of Rest $-U_{\odot}=+10.0 \mathrm{~km} \mathrm{~s}^{-1}, V_{\odot}=+5.2 \mathrm{~km} \mathrm{~s}^{-1}$, and $W_{\odot}=+7.2 \mathrm{~km} \mathrm{~s}^{-1}$ according to Binney \& Merrifield (1998) and the spin of the MW (+220 $\left.\mathrm{km} \mathrm{s}^{-1}\right)$ adopted by IAU (1986). The three components of the corrected velocity vector for each galaxy in our sample are listed in Table 2.

Finally, the coordinates of the $\mathrm{dG}$ barycentre in the galactic frame of reference are obtained (see Mateo 1998) with

$x=R_{\odot}-d \cos b \cos l$

$y=d \cos b \sin l$

$z=d \sin b$

where $R_{\odot}=8 \mathrm{kpc}$ is assumed.

The data listed in Table 2 are considered to indicate the present-day kinematical and dynamical status of each $\mathrm{dG}$ orbiting about the MW. The assumption is made that in the past no other celestial bodies (no matter whether exclusively made of baryonic matter or DM or a combination of both) by virtue of their mass, dimensions, trajectory have significantly perturbed the orbital motion of the dwarf object (Klypin et al. 1999; Blitz et al. 1999; Moore et al. 1999b).

Finally, the initial total mass of the $\mathrm{dG}$ is suitably chosen in order to get, after several Gyr of gravitational interaction with the MW and consequent mass loss by dynamical effects, an 
object with baryonic mass of about $10^{6} M_{\odot}$ as suggested by the data for dGs in the LG (Mateo 1998).

\section{The starting model for dlrrs}

The dIrr galaxy, whose dynamical structure is tested against the gravitational interaction with a large mass object, is supposed to have been formed in the past and to be made of three components: DM, gas, and stars in suitable proportions. The total mass of the galaxy is given by the sum of the three components $M_{\mathrm{G}}=M_{\mathrm{S}}+M_{\mathrm{g}}+M_{\mathrm{DM}}$ with obvious meaning of the symbols.

The rotation curves (Hoffman et al. 1996) and cosmological arguments (Mateo 1998, and reference therein) have clearly indicated that galaxies (the dwarf ones in particular) are DM dominated. Therefore we assume that $M_{\mathrm{DM}}=$ $0.90 M_{\mathrm{G}}$. Furthermore, dIrrs are known to be gas-rich, with the gaseous content almost paralleling the stellar content. We adopt here $M_{\mathrm{g}}=M_{\mathrm{s}}$.

The time scale required to build up the dIrr galaxy to the stage we are considering is not relevant here provided it is significantly shorter than the Hubble time. Furthermore, stellar activity is supposed to be still underway so that during the simulation more gas is converted into stars. Finally, let us denote with $T_{0}$ the age of the dIrr at the start of the dynamical simulation.

We approximate the dIrr galaxy with a disk. The advantage with this choice is that

(i) the structure of disk galaxies are well studied and easy to handle in the set up of the initial conditions of the orbital motion;

(ii) one of the spatial dimensions of a disk is much smaller than the other two. The vertical scale height is indeed much shorter than the horizontal scale length fixed by the plane of symmetry itself. The radius of the disk is assumed here to be equal to the typical size of a dG;

(iii) the effects of the gravitational interaction can be easily singled out. As a matter of fact, the three dimensional structure of a stable disk evolved in isolation is expected not to change under the action of self-gravity. In contrast, if under the gravitational action of an external dominant body dynamical instabilities may arise, the scale height along the vertical direction of the disk is expected to increase;

(iv) what is found with a stable disk will a fortiori occur with an irregular, much more loosely bound galaxy.

To set up the starting model we have followed Hernquist (1993a,b), whose models have been extensively proved to produce stable configurations for systems with more than one component. The disk is supposed to be made of gas and stars, whose radial mass distribution is an exponential law with a suitable scale length (Freeman 1970; Bahcall \& Soneira 1980) and vertical mass distribution is represented by isothermal layers (Spitzer 1941a,b; 1942) with scale height independent from the radial distance (van der Kruit \& Searle 1981a,b; 1982a,b).
In cylindrical coordinates the mass density profile of the disk is described by

$\rho_{\mathrm{D}}(R, z)=\frac{M_{\mathrm{D}}}{4 \pi h^{2} h_{z, \mathrm{D}}} \mathrm{e}^{-\frac{R}{h_{R, \mathrm{D}}}} \operatorname{sech}^{2}\left(\frac{z}{h_{z, \mathrm{D}}}\right)$

where $M_{\mathrm{D}}$ is the total mass of the disk, $h_{R, \mathrm{D}}$ is its radial scale length and $h_{z, \mathrm{D}}$ is its vertical scale height.

The scale height of the stars is $h_{z, \mathrm{~s}}=0.1 \mathrm{kpc}$ and their maximum vertical extension is $z_{\mathrm{s}, \max }=1.0 \mathrm{kpc}$. The scale height of the gas is $h_{z, \mathrm{~g}}=0.020 \mathrm{kpc}$ and the maximum extension of it is $z_{\mathrm{g}, \max }=0.9 \mathrm{kpc}$. The model is alike to the MW re-scaled to these dimensions (e.g. Dehnen \& Binney 1998)

The initial radius of the disk is $R_{\mathrm{D}}=6 \mathrm{kpc}$ both for stars and gas, even though it is observationally known that the gas disks could be in general more extended than stellar disks (Cote et al. 1997). No material is assumed to exist beyond this radius, therefore the maximum extensions of the gas and star distributions are the same, $R_{\mathrm{s}, \max }=R_{\mathrm{g}, \max }=6 \mathrm{kpc}$. This approximation is also sustained by numerical simulations showing that all the gas beyond the radius adopted for the stellar component is soon lost during the first evolutionary stages $\left(T_{0}+T \leq 1.5 \mathrm{Gyr}\right)$ with no consequence for the future dynamical structure of the satellite galaxy (see Mayer et al. 2001a and below).

Following Binney \& Tremaine (1994), and Athanassoula \& Sellwood (1986), a disk is stable to all axisymmetric perturbations if the Toomre $(1963,1964)$ parameters

$Q_{\mathrm{s}}=\frac{\sigma_{R}(R) \chi(R)}{3.36 G \Sigma(R)}>2$

for the stars and/or

$Q_{\mathrm{g}}=\frac{\sigma_{R}(R) \chi(R)}{\pi G \Sigma(R)}>2$

for the gas are satisfied. In relations (9) and (10) $\sigma_{R}$ is the radial velocity dispersion, $\chi$ is the epicyclical frequency, and $\Sigma$ is the surface density of the disk. The Toomre relations are commonly accepted conditions in many studies of galactic dynamics (e.g. Mayer et al. 2001b; Huang \& Carlberg 1997; Hernquist 1993a,b). They are fully adequate for our aims. Since we do not intend to investigate the internal structure (bars and spiral arms) of a transient disk-shaped galaxy (see. e.g. Jerjen et al. 2001; De Rijcke et al. 2001; Barazza et al. 2002) we have not considered more sophisticate studies (e.g. Jog 1996; Jog 2000; Romeo 1992; Pichon \& Cannon 1997). The radial profile of the Toomre parameters of our model are shown in Fig. 1. They are similar to those of Mayer (2001a).

Finally, a spherical halo of DM has been added to the model galaxy. The radial dependence of the density and gravitational potential profiles of the halo are supposed to be globally represented by the phenomenological density profile (Hernquist 1993a) (but see e.g. Widrow 2000) ${ }^{1}$.

$$
\begin{aligned}
\rho_{\mathrm{H}}(r) & =\frac{M_{\mathrm{H}}}{2 \pi^{\frac{3}{2}}} \frac{\alpha}{r_{\mathrm{c}}} \frac{\exp \left(-\frac{r^{2}}{r_{\mathrm{c}}^{2}}\right)}{r^{2}+\gamma^{2}} \\
\alpha & =\frac{1}{1-\sqrt{\pi} \cdot q \cdot \exp \left(q^{2}\right)(1-\operatorname{erf}(q))}
\end{aligned}
$$

1 The Hernquist (1990) profile has been also considered with no significant difference in the final results. 


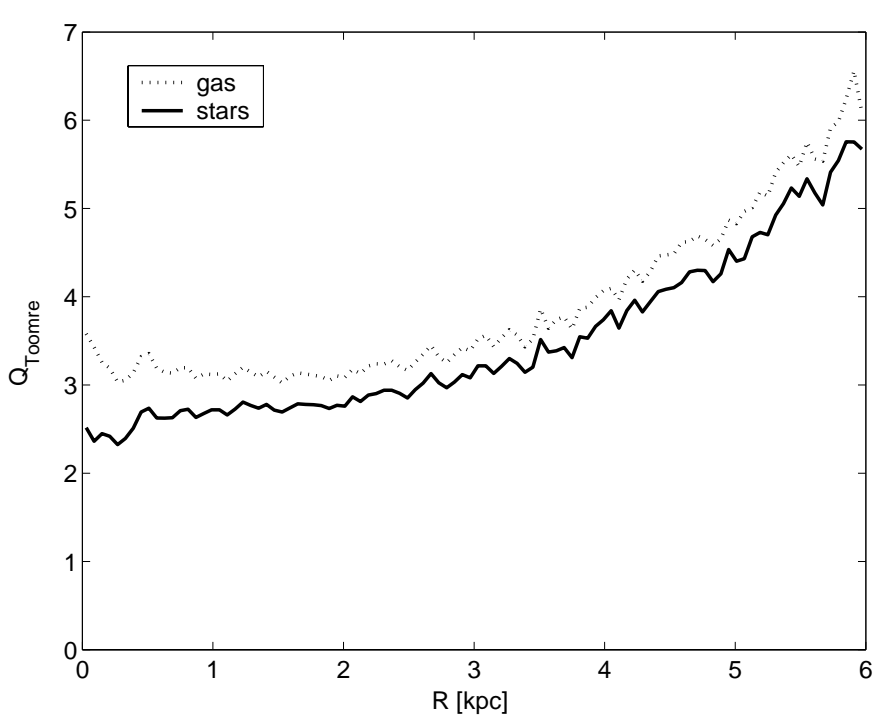

Fig. 1. The Toomre parameters for gas and stars as function of the radial distance in the initial disk galaxy. Both parameters indicate that the structure is stable against the bar instability.

where $q=\frac{\gamma}{r_{\mathrm{c}}}, \gamma=1 \mathrm{kpc}$ is the core radius of the halo, and $r_{\mathrm{c}}=6 \mathrm{kpc}$ is the cut-off radius assumed for the starting model of the $\mathrm{dG}$.

The set up of the velocity ellipsoid of initial speeds for the disk and halo is based on the models by Lewis \& Freeman (1989), van der Kruit \& Searle (1981a,b) and the use of the Collisionless Boltzmann Equations and its lowest order moments (Hernquist 1993a). The radial profiles of rotational velocity and velocity dispersion of the initial configuration of the dwarf disk galaxy are shown in Figs. 2 and 3, respectively. In a scenario in which a galaxy forms for the cooling of gas within DM halos (White \& Rees 1983) the visible matter settles onto a disk rotating in centrifugal equilibrium with the halo. Therefore, contrary to Hernquist (1993b), we have artificially imposed onto DM halo a rotational velocity supposedly causing the rotation of the baryonic matter. This approach was preferred to the classical one of adopting rigid rotation with a low spin parameter for the DM halo (Kawata 2001) which would require a significant period of time to establish the profiles of circular velocities shown in Fig. 2. Finally, as explained in more detail below, we have not considered the particular evolutionary and cosmological history that led to the initial model of our $\mathrm{dG}$ (i.e. previous formation mechanisms).

The model galaxy we are considering has $M_{\mathrm{G}}=4.9 \times$ $10^{8} M_{\odot}$, which in turn splits in $4.41 \times 10^{8} M_{\odot}$ of DM (described by 7000 particles), $2.45 \times 10^{7} M_{\odot}$ of stars (described by 5000 particles), and $2.45 \times 10^{7} M_{\odot}$ of gas (described by 5000 particles). All the key structural parameters of the initial model of the satellite galaxy are summarized in Table 3.

\section{Evolution of a dlrr in isolation}

Having assigned the initial conditions, the evolution of the dIrr (disk) galaxy in isolation has been followed by means of the $N$-body Tree-SPH code of Carraro et al. (1998), Buonomo et al. (2000) in the last version described by Lia et al. (2002). The

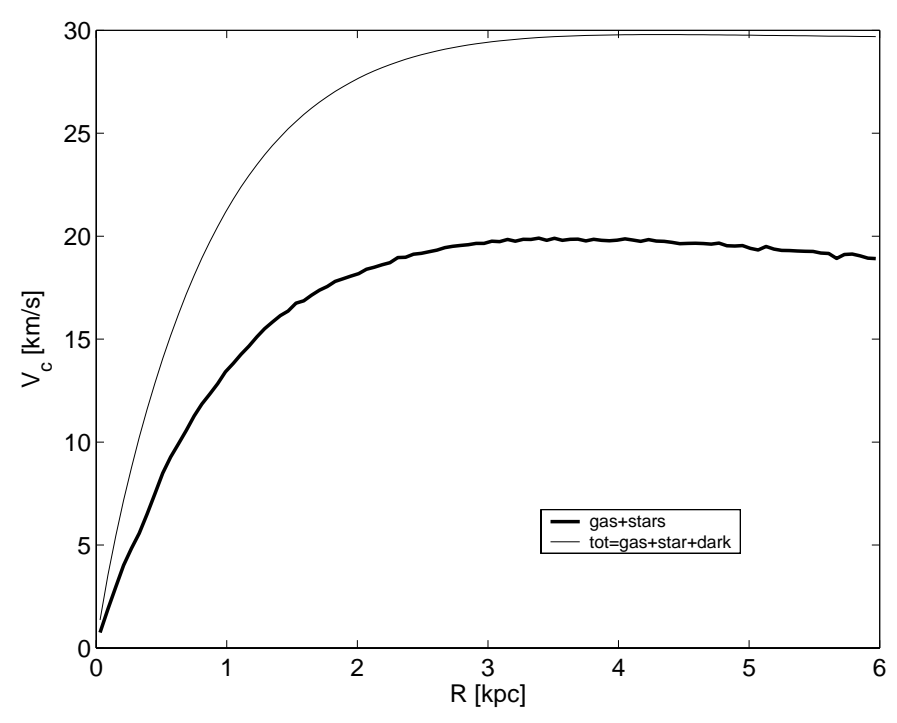

Fig. 2. The radial profiles of the circular velocities of gas and stars lumped together (thick line) and DM (thin line). The profile of the disk components calculated in the standard way does not significantly differ from the one obtained with the Bessel approximation for the potential of thin disks. This agrees with the Toomre condition against the bar instability. The flatness of the profiles at large radii is due to the massive DM halo.
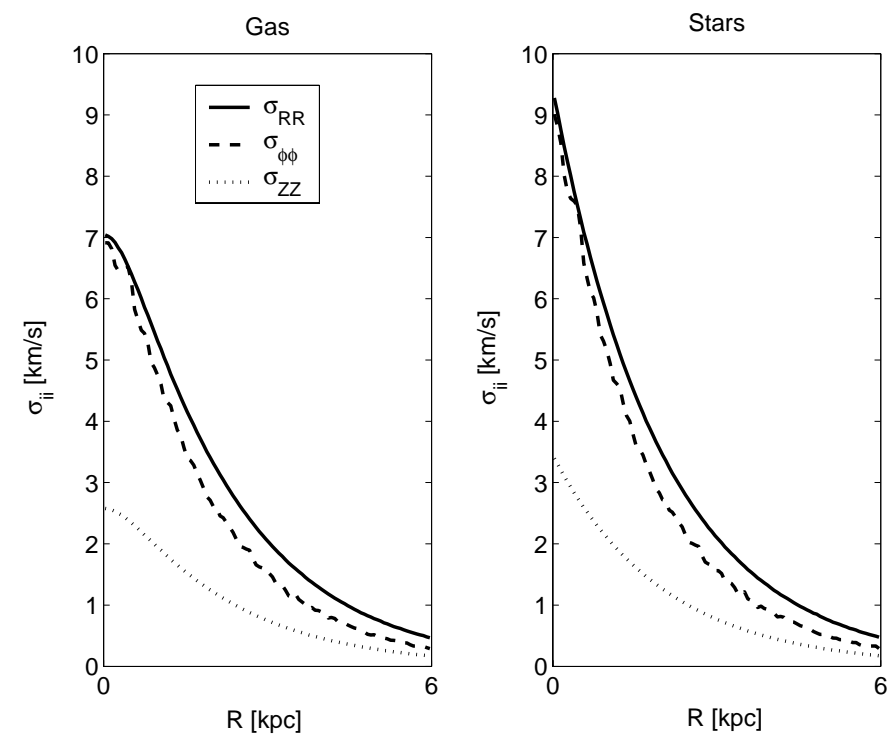

Fig. 3. Radial profiles of the three components of the velocity dispersion for the gas and stars in the initial disk galaxy. The only the nonzero diagonal terms of the velocity tensor for the normal distribution function are plotted.

code includes star formation, cooling, thermal conduction, energy feedback by Type I and II supernovae, stellar winds and ultraviolet flux from massive stars, and chemical enrichment. The code calculates the gravitational interactions with the hierarchical tree algorithm of Barnes \& Hut (1986) assuming a tolerance parameter $\theta=0.8^{\circ}$ and expanding the tree nodes to quadrupole order. The gas particles are treated according to the SPH technique (Lucy 1977; Gingold \& Monaghan 1977), in which the spherical kernel of Monaghan \& Lattanzio (1985) is adopted with a smoothing parameter $h$ variable in space and 
Table 3. Summary table of the structural parameters of the initial model for the dG (a disk) launched in orbit around the MW at the age $T_{0}$.

\begin{tabular}{cccc|ccccc}
\hline \hline \multicolumn{7}{c|}{ Masses $\left(10^{7} M_{\odot}\right)$} & \multicolumn{5}{c}{ Mass resolution $\left(10^{4} M_{\odot}\right)$} \\
\hline$M_{\mathrm{T}}$ & $M_{\mathrm{g}, \mathrm{T}}$ & $M_{\mathrm{s}, \mathrm{T}}$ & $M_{\mathrm{D}, \mathrm{T}}$ & $\Delta M_{\mathrm{g}}$ & $\Delta M_{\mathrm{s}}$ & $M_{\mathrm{DM}}$ & \\
\hline 49.0 & 2.45 & 2.45 & 44.1 & 0.49 & 0.49 & 6.30 & \\
\hline \hline \multicolumn{8}{c}{ Scale parameters $(\mathrm{kpc})$} \\
\hline$h_{z, \mathrm{~s}}$ & $z_{\mathrm{s}, \max }$ & $h_{z, \mathrm{~g}}$ & $z_{\mathrm{g}, \max }$ & $R_{\mathrm{D}, \max , \mathrm{s}}$ & $R_{\mathrm{D}, \max , \mathrm{g}}$ & $\gamma_{\mathrm{H}}$ & $r_{\mathrm{c}}$ & $R_{\mathrm{H}, \max }$ \\
\hline 0.100 & 1.000 & 0.020 & 0.900 & 6.000 & 6.000 & 1.000 & 6.000 & 12.000 \\
\hline
\end{tabular}

time (Benz 1990; Nelson \& Papaloizou 1994). The equations of motions are solved at successive time-steps according to the modified leap-frog method of Katz et al. (1996).

As already mentioned, no attempt is made to derive, starting from cosmological conditions, e.g. Bertschinger (2001), the structure of the disk galaxy up to the age $T_{0}$. By adopting this simplified view of the problem, a great deal of computational time is saved without losing in generality. Furthermore this allows us to treat only collisionless effects of the gas particles in the set up of the initial model, thus avoiding more complicate schemes in which collisional gas clouds effects are present, see for instance Bekki \& Chiba (2000, 2001).

As far as the physical ingredients we have adopted to build the galaxy model are concerned, we briefly summarize here the formula for the rate of star formation (SF). This follows the Schmidt (1959) law

$\frac{\mathrm{d} \rho_{\star}}{\mathrm{d} t}=-\frac{\mathrm{d} \rho_{\mathrm{g}}^{K}}{\mathrm{~d} t}=-\frac{c_{\star} \rho_{\mathrm{g}}^{K}}{t_{\mathrm{g}}}$

where $c_{\star}$ is the so-called dimensionless efficiency of SF for which we adopt $c_{*}=1.0, t_{\mathrm{g}}$ is a characteristic time scale, and $\kappa=1.3$. The time scale $t_{\mathrm{g}}$ is chosen to be the maximum between the cooling, $t_{\mathrm{cool}}$, and the free-fall, $t_{\mathrm{ff}}$, timescales. The cooling time-scale is derived from the equation $t_{\text {cool }}=E /\left|\dot{E}_{\mathrm{H}}-\Lambda_{\mathrm{C}} / \rho_{\mathrm{g}}\right|$, where $E, \dot{E}_{\mathrm{H}}$, and $\Lambda_{\mathrm{C}}$ are the current thermal energy per unit mass of gas, the heating rate per unit mass of gas by all possible sources, and the cooling rate (in $\mathrm{erg} \mathrm{s}^{-1} \mathrm{~cm}^{-3}$ ), respectively. For more details on $E_{\mathrm{H}}, \Lambda_{\mathrm{C}}$, and associated cooling time-scales see Chiosi et al. (1998) and Carraro et al. (1998). The free-fall time is $t_{\mathrm{ff}}=(1 / 4 \pi G \rho)^{0.5}$, where $\rho$ is the total mass density (gas + stars $+\mathrm{DM})$. In addition to this, following Katz (1992) and Navarro \& White (1993), a gas particle is able to form stars if the conditions $t_{\text {sound }}>t_{\text {ff }}$ and $t_{\text {cool }} \ll t_{\text {ff }}$ are met, where $t_{\text {sound }}$ is the sound crossing time.

The numerical results show that the model for the dIrr (disk) galaxy we have adopted is dynamically stable when evolved in isolation under the sole action of self-gravity during a period of time comparable to three complete revolutions about the MW of a satellite like Sculptor or equivalently a sizable fraction of the Hubble time. The schematic structure of the dIrr (disk) galaxy evolved in isolation is shown in Fig. 4 for the age $T_{0}$ (initial model) and Fig. 5 for the age $T_{0}+6 \mathrm{Gyr}$.

\subsection{Analysis of the isolated $d G$ after 6 Gyrs}

The four panels of Fig. 5 summarize the structure of the isolated dG after 6 Gyr of evolution. The top left panel shows the projection onto the $X-Y$ plane of the gas (small circles), stars (crosses), and DM (small dots) particles, whereas the top right panel displays the projection onto the $X-Z$ plane of the gas (small circles) and stars (crosses) particles, DM particles have been omitted for the sake of clarity. The two dotted and two heavy dashed lines drawn in the top right panel of Fig. 5 show the $1 \times \sigma_{R}$ and $1 \times \sigma_{Z}$ horizontal and vertical distribution of baryonic matter, respectively. It turns out that about $70 \%$ of the baryonic mass is contained within a cylinder of $1.62 \mathrm{kpc}$ radius and $0.34 \mathrm{kpc}$ height. To better show the morphological evolution of the disk galaxy, in the left panel of Fig. 5 we present a 3-D picture of the whole galaxy (gas, stars and DM), whereas in the right bottom panel we display a slice of it limited to gas and stars.

It is soon evident that some structural evolution has taken place since the start of the simulation at $T_{0}$, (see the corresponding panel in Fig. 4). While the radial dimensions have remained unchanged (see the star mass profiles in Fig. 6 below), the thickness of the stellar disk along the $Z$-axis has naturally increased, leading to the formation of a thick disk, whose vertical extension is about $\simeq 1.5 \mathrm{kpc}$ height (e.g. Villumsen 1985). The gas particles, whose total mass has been decreased by star formation, are mostly located within in the inner potential well of the structure keeping substantially unchanged cinematic and morphology. However, a small fraction of these are pushed away from the plane of the disk to large distances under the action of heating. Finally, after the initial period of about $1 \mathrm{Gyr}$, the star formation activity falls down to low levels as shown in the top panel of Fig. 23 to be discussed later. Despite those morphological changes with respect to the initial model, for the purposes of this study we may conclude that our test $\mathrm{dG}$ has remained a disk object over 6 Gyr of evolution.

The differential mass distribution versus the radial distance (mass per $\Delta R$ bin) is shown in Fig. 6 both for gas and stars and the ages $T_{0}$ and $T_{0}+6 \mathrm{Gyr}$. The following remarks are worth noting: (i) as expected a large fraction of the gas is converted into stars; (ii) star formation takes place preferentially within the central region of about $2 \mathrm{kpc}$ radius, into which a significant amount of gas sinks from the outermost regions to be converted into stars; (iii) the external regions (beyond about $2 \mathrm{kpc}$ ) remain essentially untouched by the star formation process (the stellar profile remains constant); (iv) finally, there is no drift of new born stars toward the center in compliance with the circular nature of the orbits.

The rotation curves for the gas, stars and DM at the age $T_{0}+$ 6 Gyr are plotted in Fig. 7 to be compared to the starting values 

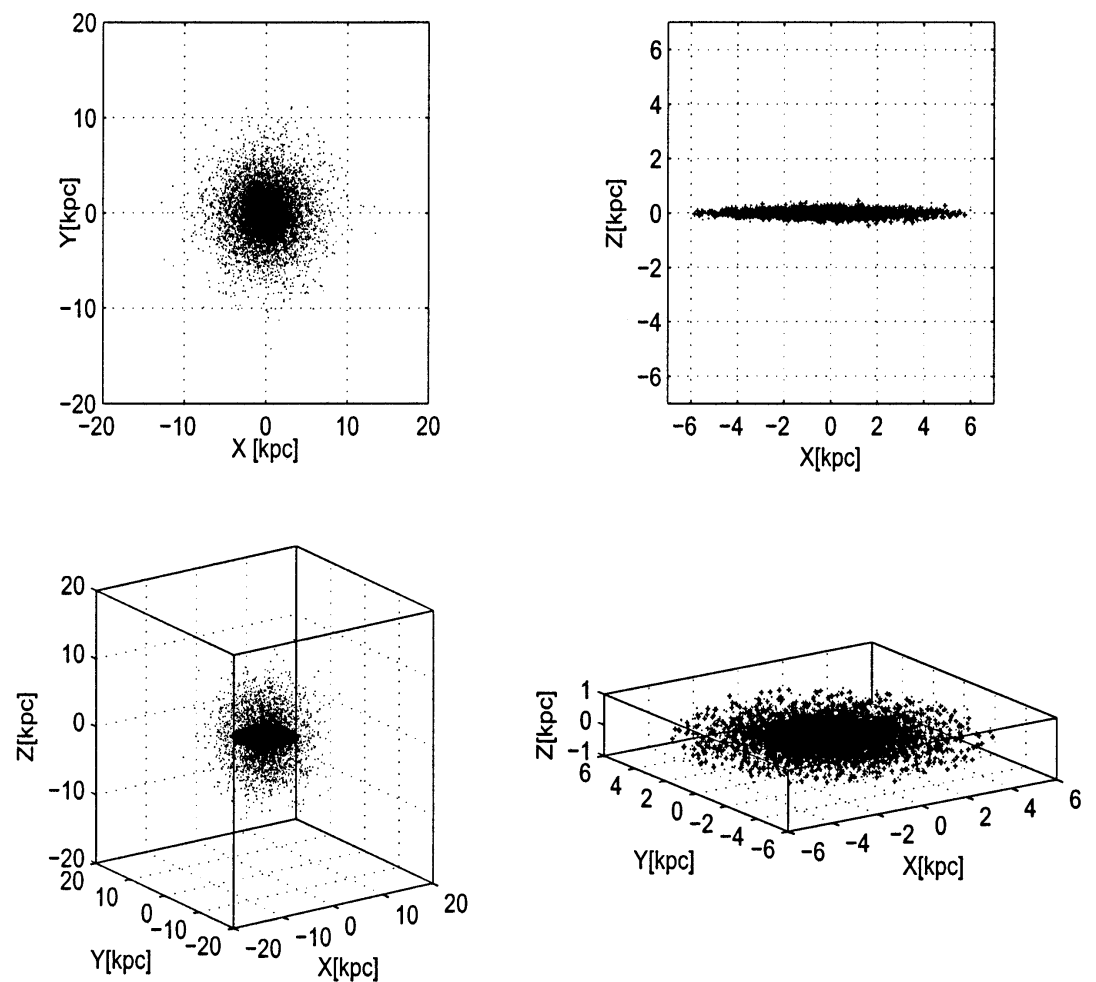

Fig. 4. The structure of a disk galaxy, made of stars, gas and DM, at the beginning of the dynamical simulation, i.e at the age $T_{0}$. In all the panels, small dots are for DM, crosses for stars, and small circles for gas. For the sake of clarity, the DM particles are plotted only in the left panels and not in the right panels. The top panels show the projections onto the $X-Y$ and $X-Z$ planes. The bottom left panels is the 3-D view of the galaxy from $38^{\circ}$ azimuth and $12^{\circ}$ elevation. The bottom right panel is a $3 \mathrm{D}$ blow-out of the central region of the disk seen from $-34.5^{\circ}$ azimuth and $16^{\circ}$ elevation. In this region of about $0.8 \mathrm{kpc}$ thickness $90 \%$ of the gas and star mass is contained.
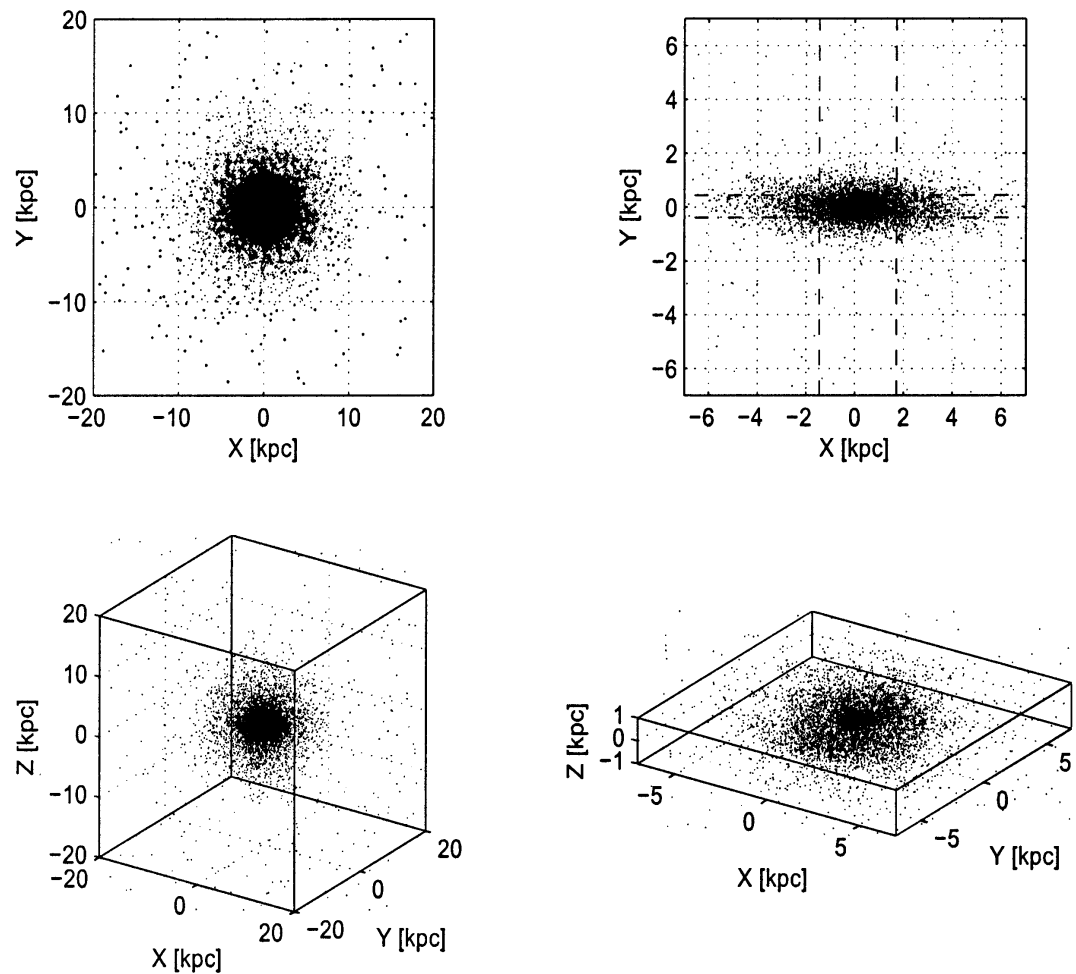

Fig. 5. The same as in Fig. 4 but 6 Gyr after the age $T_{0}$. Although the galaxy has undergone some changes in the radial dimensions and thickness, still a disk-like structure has remained, fully adequate to our purposes. The vertical dotted lines and the horizontal dashed lines drawn in the top right panel correspond to the $1-\sigma$ limit for the density distribution of gas and stars. 


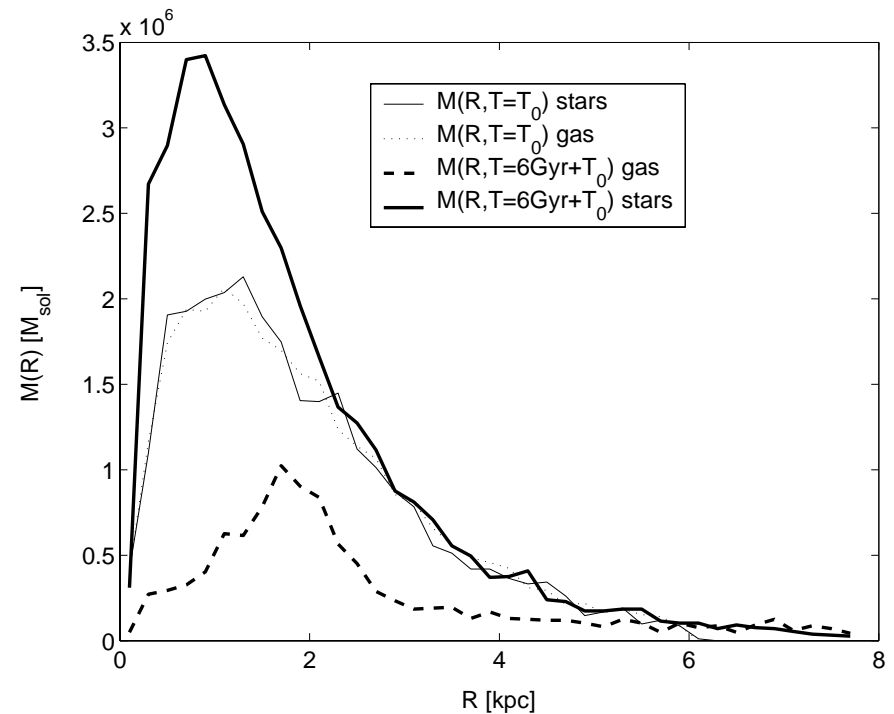

Fig. 6. The differential mass distribution as a function of the radial distance (mass per $\Delta R$ bin). The thin a solid and dotted lines represent the mass in stars and gas, respectively, for the initial model at the age $T_{0}$. The thick solid and dashed lines are the same but for the model at the age $T_{0}+6 \mathrm{Gyr}$. The origin of the radial coordinate is the galaxy barycentre.

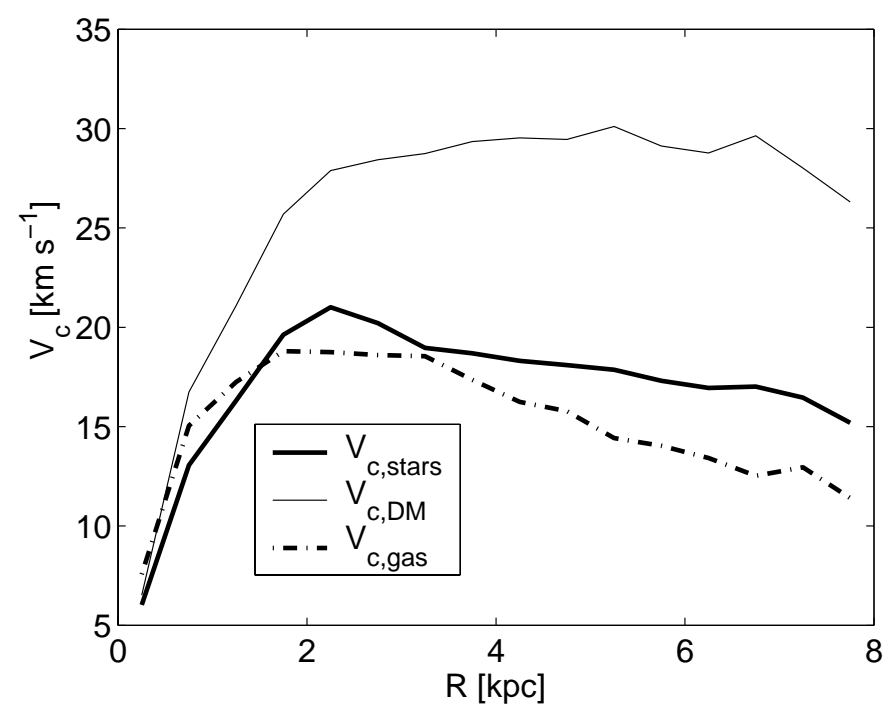

Fig. 7. Radial profiles of the circular velocities of the gas (dasheddotted line), stars (thick solid line), and DM (thin solid line) in the model at the age $T_{0}+6 \mathrm{Gyr}$. As expected, the profiles for the baryonic matter remain sufficiently flat in the outermost regions dominated by DM. These profiles can be compared to those of the initial model shown in Fig. 2.

of Fig. 2. Finally, assuming the mass to $B$-light ratio $M / L_{B}=2$ (Bottema 1997) we may derive the surface brightness profile. This is shown in Fig. 8 for the two ages $T_{0}$ and $T_{0}+6 \mathrm{Gyr}$ clearly indicating that the surface brightness profile has remained unchanged.

An important remark to be made here concerns the conservation laws. The problem and the code obey the laws of conservation of the energy and total number of particles. However, even in the case of evolution in isolation - the problem will

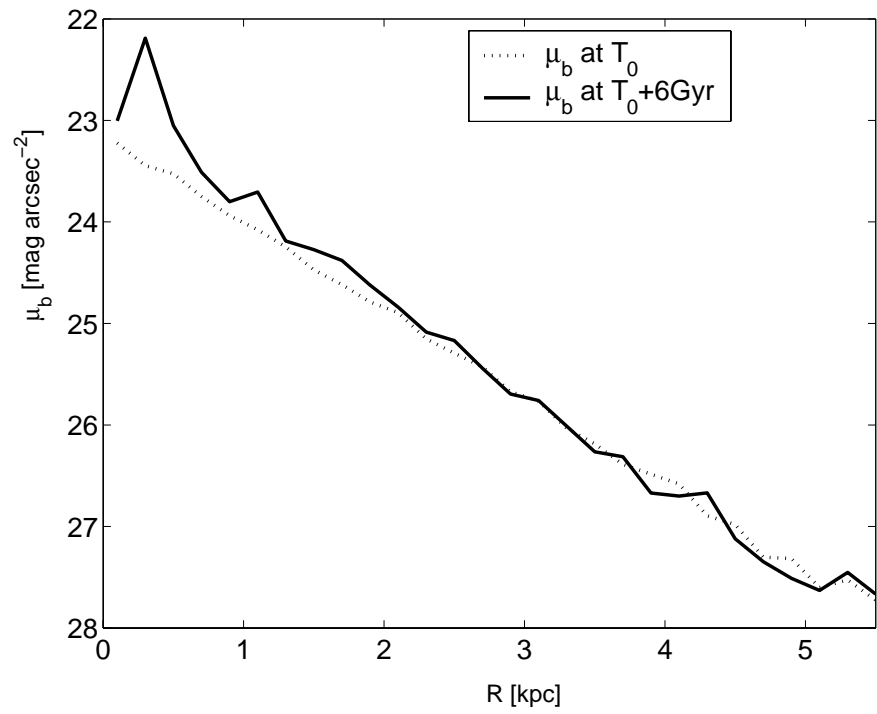

Fig. 8. The surface brightness profile. The solid line is for the model with age $T_{0}+6 \mathrm{Gyr}$, whereas the dotted line is for the initial one. As expected no significant change occurs.

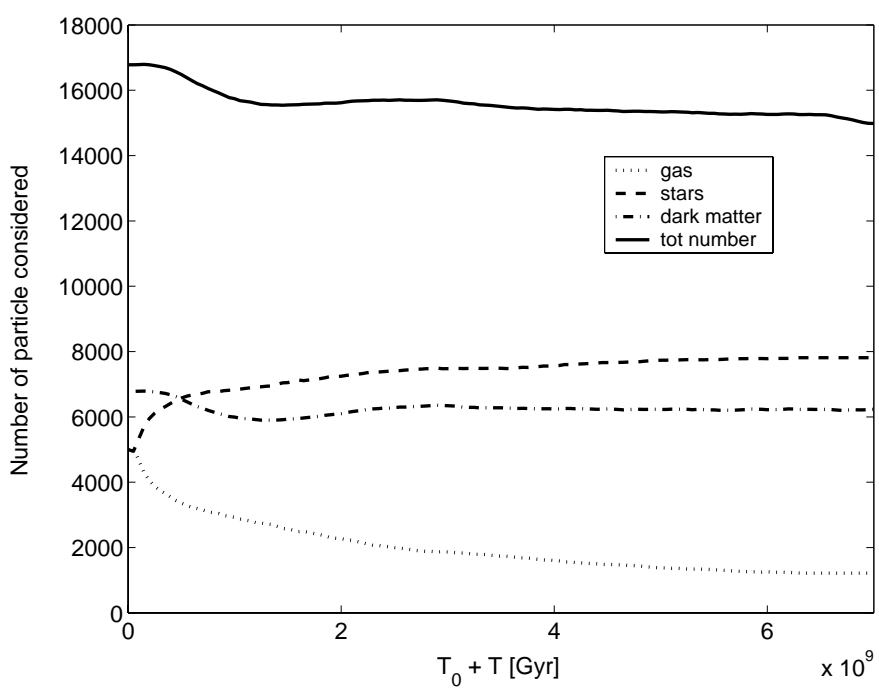

Fig. 9. The number of gas (dotted line), star (dashed line), and DM (dotted-dashed line) particles as a function of time in the model galaxy. The thick solid line is the same but for the total number of particles. See the text for details.

be more severe in the case of a satellite interacting with a host galaxy (see below) - a certain number of particles and amount of mass in turn are lost by the system because they acquire sufficient energy by interactions to overwhelm the gravitational potential and leave the galaxy as galactic wind (Chiosi \& Carraro 2002). Therefore we need to limit our analysis of the galaxy properties to a certain volume which is not affected by this phenomenon and yet contains sufficient information. The starting volume under consideration is the one inside a box containing at least $90 \%$ of baryonic mass.

The decrease in the total number of particles and the differential mass distribution is shown in Figs. 9 and 10, respectively.

While the transformation of the gas into stars easily explains the decrease in the total number of gas particles and 


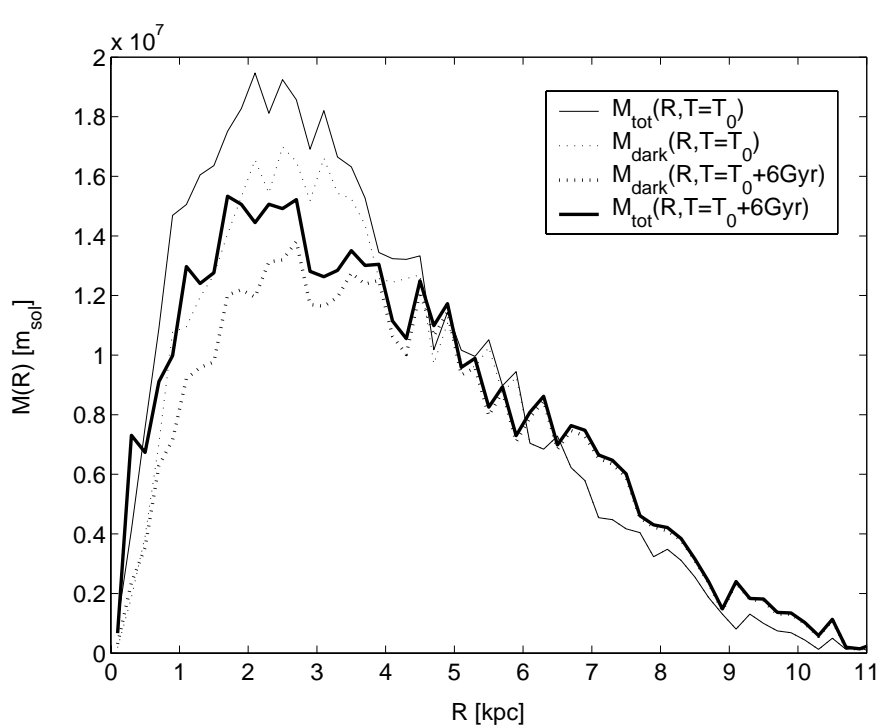

Fig. 10. Radial profiles of the differential distribution of mass (mass per $\Delta R$ bin) for the initial model (thin lines) and the model of $T_{0}+6 \mathrm{Gyr}$ age (thick lines). The dotted lines are for DM, the solid lines for the total mass (gas+stars+DM). See the text for details.

the corresponding increase in the total number of star particles (see Fig. 9) there is also a low decrease in the total number of DM particles (see once again Fig. 9) at least in the region under examination. They are either pushed too far away or even lost. The mass profiles shown in Fig. 6 simply mirror this situation. Some $11 \%$ of the mass is subtracted to the central body of the disk galaxy.

\subsection{Numerical stability}

The numerical results of the case evolved in isolation is particularly important to check the numerical stability of the model. Too massive halo particles may cause an artificially heating of halo structure (Moore et al. 1996a) because of the two body relaxation effect in $N$-body simulations. The same may happen with a dG disk galaxy, especially in its central regions with the highest mass concentration. A viable solution of the problem is to adopt a high number of DM particles thus reducing their to specific gravitational attraction on disk particles. As shown in Fig. 9 this allows us to the disk structure nearly unchanged, to avoid artificial evaporation of halo particles, and to secure the numerical stability of the results we are interested in (Knebe et al. 2000).

\section{Evolution of a dlrr orbiting around the MW}

The dIrr (disk) galaxy is now supposed to orbit around the MW whose presence is simply reduced to a force field generated by a suitable, constant gravitational potential at the coordinate origin and with no rotation. Furthermore, following Peebles (1989) we assume that the orbits of the satellite dwarf galaxy are always inside the DM Halo of the MW.

In this study we neglect dynamical friction which is one of the fundamental and most investigated process that can characterize the orbital decay of the dG toward the center and cause the loss of orbital energy and angular momentum. Since the pioneering studies of Chandraseckhar on stellar dynamics (Chandraseckhar 1941a-d, 1943), that first analyzed the effect of dynamical friction restricted to an infinite, uniform, non-self-gravitating stellar backgrounds, many efforts are been made to understand the impact of this physical process on the global dynamical behaviour of two interacting galaxies with somewhat contradictory results. The addressed questions concerned the local or large scale nature of dynamical friction and its effects on a static center of mass and on the auto-gravity of the main galaxy about which the satellite is orbiting (Spitzer \& Hart 1971; White 1976, 1978, 1983; Tremaine 1975; Quinn \& Goodman 1986; Bontekoe \& Albada 1978; Lin \& Tremaine 1983; Weinberg 1989; Hernquist \& Weinberg 1989; Prugniel \& Combes 1992; Leeuwin \& Combes 1997; Cora et al. 1997).

Recent progress has been made by means of the linear response theory (Colpi \& Pallavicini 1998; Colpi 1998) which takes into account simultaneously and analytically the gravitational wake, the tidal deformation and the shift of the barycentre of the primary galaxy, thus unifying the local and large-scale interpretation of dynamical friction and providing good agreement with the $N$-body simulations (Colpi 1999).

In the specific case of a dG, it can be shown that dynamical friction can be neglected because the orbital decay time scales would be much longer than the Hubble time. The reason is the low mass of the satellite (e.g. $4.9 \times 10^{8} M_{\odot}$ ) compared to that of the MW $\left(10^{11} M_{\odot}\right)$ and the additional delay introduced by the tidal stripping (Colpi 1999; Mayer et al. 2001a,b).

\subsection{Gravitational potential of the Milky Way}

\subsubsection{The Halo}

Various non-rotating potentials of the DM Halo (supposed to be spherical for simplicity) have been tested and implemented in the $N$-body Tree-SPH code: Newtonian, Plummer, Allen \& Santillán, and logarithmic. The choice has finally been restricted to the potential of Allen \& Santillán (1991) who improved the previous one by Allen \& Martos (1986). The potential we have chosen is generated by the spherical distribution of mass given by

$M_{\mathrm{HMW}}(R)=\frac{M_{\mathrm{HMW}}\left(\frac{R}{R_{\mathrm{HMW}}}\right)^{\gamma}}{1+\left(\frac{R}{R_{\mathrm{HMW}}}\right)^{(\gamma-1)}}$

where $M_{\mathrm{HMW}}(R)$ is the mass profile of the MW Halo, $M_{\mathrm{HMW}}$ and $R_{\mathrm{HMW}}$ are its total mass and scale radius, respectively, and $R$ is the galacto-centric radial distance. The best fit to the observational data is obtained assuming $M_{\mathrm{HMW}}=1.07 \times$ $10^{11} M_{\odot}, R_{\mathrm{HMW}}=12 \mathrm{kpc}$, and $\gamma=2.02$.

The gravitational potential corresponding to the above mass distribution is

$\Phi_{\mathrm{HMW}}(R)=-\frac{G M_{\mathrm{HMW}}(R)}{R}-\int_{R}^{R_{\mathrm{HMW}, T}} \frac{G}{R^{\prime}} \frac{\mathrm{d} M_{\mathrm{H}}\left(R^{\prime}\right)}{\mathrm{d} R^{\prime}} \mathrm{d} R^{\prime}$

where $R_{\mathrm{HMW}, T}$ is the maximum extension of the Halo for which we adopt $R_{\mathrm{HMW}, T}=200 \mathrm{kpc}$. 
Finally, the explicit analytical expression for the gravitational potential of the MW Halo is

$\Phi_{\mathrm{HMW}}(R)=-\frac{G M_{\mathrm{HMW}}(R)}{R}-\frac{G M_{\mathrm{HMW}}}{(\gamma-1) R_{\mathrm{HMW}}}$

$\times\left[-\frac{\gamma-1}{1+\left(\frac{R}{R_{\mathrm{HMW}}}\right)^{\gamma-1}}+\ln \left(1+\left(\frac{R}{R_{\mathrm{HMW}}}\right)^{\gamma-1}\right)\right]_{R}^{R_{\mathrm{HMW}, T}}$.

\subsubsection{The disk}

The potential used for the disk of the MW is taken from Miyamoto \& Nagai (1975)

$$
\Phi_{\mathrm{DMW}}(R, z)=-\frac{G M_{\mathrm{DMW}}}{\sqrt{R^{2}+\left(R_{\mathrm{DMW}}+\sqrt{z^{2}+z_{\mathrm{DMW}}^{2}}\right)^{2}}}
$$

where $R$ is the galacto-centric radial coordinate, $z$ is the vertical coordinate above the plane of the disk, $M_{\mathrm{DMw}}, R_{\mathrm{DMW}}$ and $z_{\text {DMw }}$ are the total mass, the radial scale length, and the vertical scale height of the disk, respectively. The following values are adopted $M_{\mathrm{DMW}}=8.56 \times 10^{10} M_{\odot}, R_{\mathrm{DMW}}=5.31 \mathrm{kpc}$, and $z_{\text {DMW }}=0.25 \mathrm{kpc}$.

Preliminary test calculations have shown that the presence of the disk does not significantly affect the orbits and the inner dynamical structure of the satellite galaxy.

\subsubsection{The Bulge}

The contribution of the Bulge to the total gravitational potential of the MW is not taken into account. Although the Bulge contain a sizable fraction of the total mass of the Galaxy its radius is much smaller than the typical orbital radius of the satellite, so that it would scarcely affect the effective gravitational potential at those distances (Mayer et al. 2001a).

\subsubsection{The total gravitational potential}

It may be of interest to examine in some detail how the presence of the disk would affect the otherwise spherically symmetric total gravitational potential. To this aim, we introduce the total gravitational potential $\Phi_{\mathrm{T}}(X, Y, Z)=\Phi_{\mathrm{DMW}}(X, Y, Z)+$ $\Phi_{\mathrm{HMW}}(X, Y, Z)$ (the contribution from the Bulge has already been neglected) and define the scalar field $\Psi(X, Y, Z)=$ $\Phi_{\mathrm{HMw}}(X, Y, Z) / \Phi_{\mathrm{T}}(X, Y, Z)$. Two sections of the scalar field, i.e. along the plane of the Disk and perpendicular to it, are shown in Fig. 11. The center of the MW coincides with the center of the coordinates. The scalar field varies from 0 to 1 and the values assumed at different distances and positions with respect to the Galactic center are indicated according to the color code shown by the vertical bar. The filled circles connected by the solid line show the 3D-view of the orbit of the satellite galaxy that we are going to consider in our simulation (see Fig. 12 below). It is soon evident that the orbit intersects regions of the scalar field whose values are $\Psi \simeq 1$. This means that the orbit is basically driven by the spherical potential of the sole Halo.

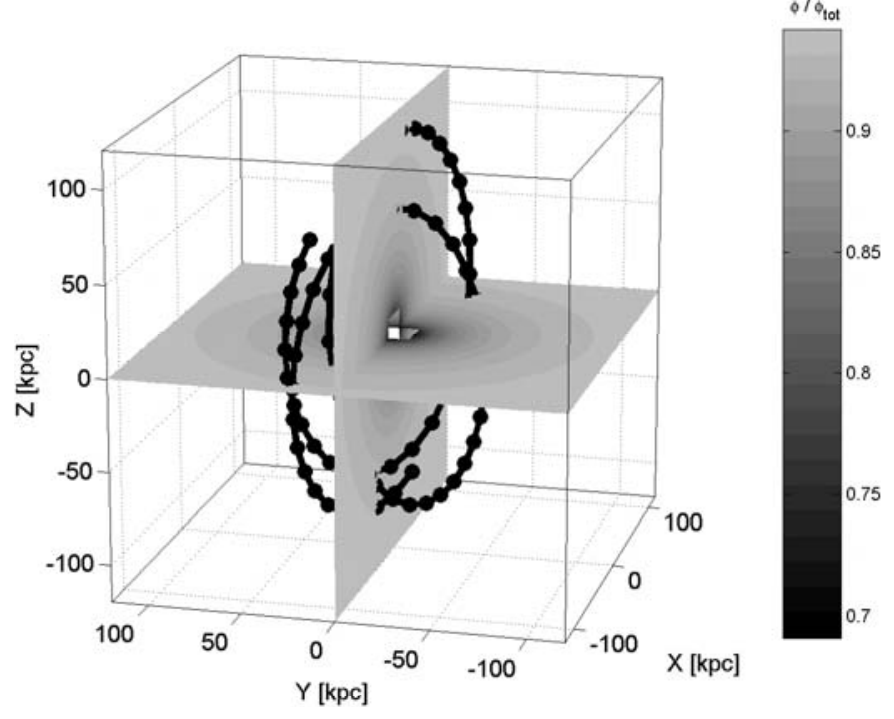

Fig. 11. 3D-view of the gravitational potential of the MW as represented by the scalar field $\Psi(X, Y, Z)=\Phi_{\mathrm{HMw}}(X, Y, Z) /\left[\Phi_{\mathrm{DMw}}(X, Y, Z)+\right.$ $\left.\Phi_{\mathrm{HMW}}(X, Y, Z)\right]$, where $\Phi_{\mathrm{DMw}}(X, Y, Z)$ and $\Phi_{\mathrm{HMw}}(X, Y, Z)$ are the Disk and Halo components, respectively. Two intersections (parallel and perpendicular to the plane of the Galactic Disk) are shown for the sake of clarity. The center of the coordinates coincides with the center of the MW. The color code shown in the vertical bar, corresponds to different values of $\Psi(X, Y, Z)$. The orbit of the satellite galaxy (Sculptor in our simulation) is shown by the solid line in steps of $0.1 \mathrm{Gyr}$ (filled circles).
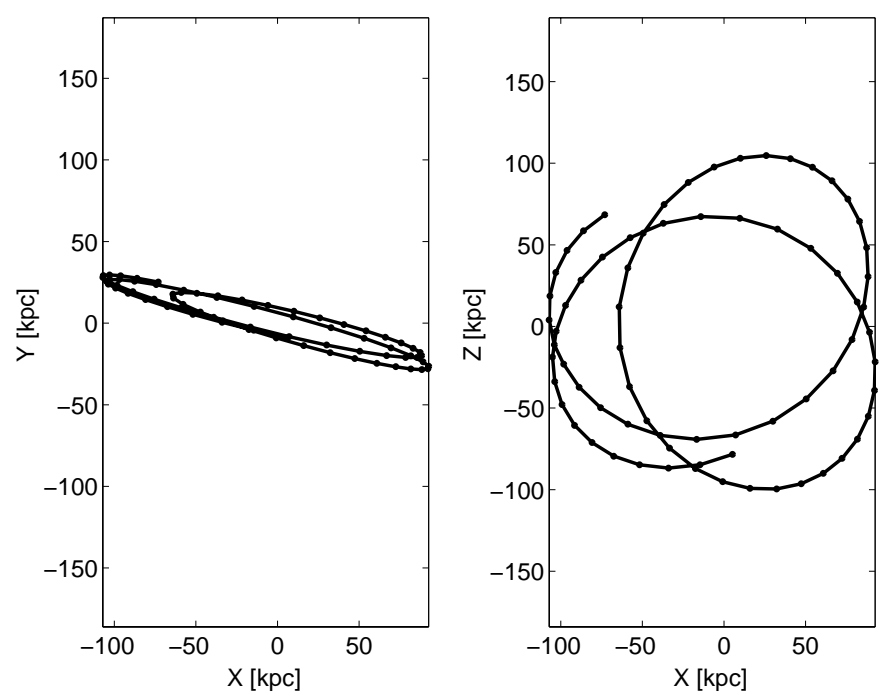

Fig. 12. Orbit of the satellite galaxy (Sculptor) during 6 Gyr after $T_{0}$ projected onto the $X-Y$ plane (left panel) and the $X-Z$ plane (right panel). The orbit is calculated in time steps of $0.1 \mathrm{Gyr}$ from $T_{0}$ to $T_{0}+6 \mathrm{Gyr}$.

At the distances reached by the satellite (Sculptor in our simulation) during its orbital motion around the MW (see Fig. 13 below), the presence of the Disk is only a minor, almost negligible, contribution to the total gravitational potential. 


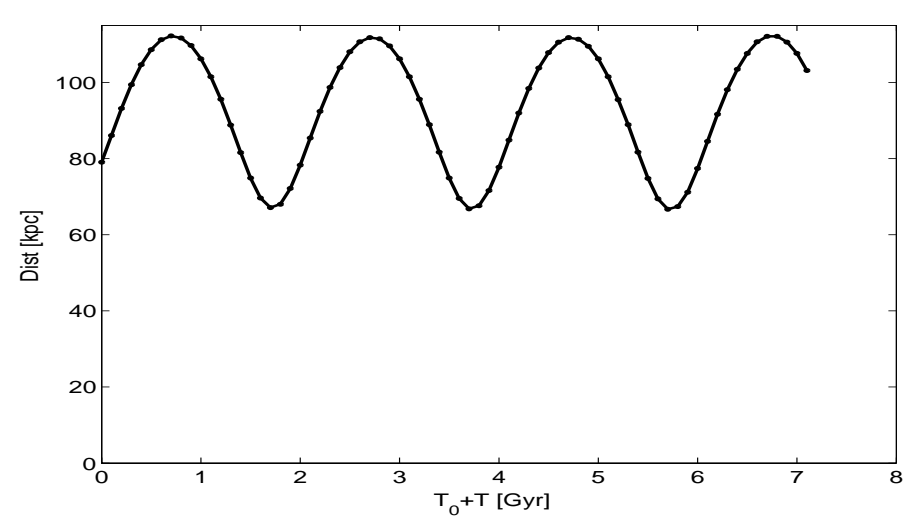

Fig. 13. Distance of the dG (Sculptor) to the MW as function of time. As expected there, the distance remains constant during the time interval of $6 \mathrm{Gyr}$ after $T_{0}$. The galactic apo-center and peri-center are about 112 and $67 \mathrm{kpc}$, respectively. The mean distance of the dG to MW is about $90 \mathrm{kpc}$.

\subsection{Choosing the dwarf galaxy}

For the purposes of this study, the choice of the satellite galaxy to simulate and to follow during its dynamical and evolutionary history is not trivial. As already anticipated, chief aim of this analysis is to check whether morphological evolution of the satellite caused by its dynamical interaction with the MW is possible. The transformation of a dIrr into a dSph is the gool.

Very distant dGs are discarded, because many passages at the peri-galactic would be required to detect effects that are expected to be small. It might occur that time scales longer than the Hubble time would be required to get a sizable effect. Examples of this situation are Draco and Ursa Minor (see the entries of Tables 1 and 2). To be fully explored those cases would likely require a large amount of computing time that perhaps is not worth being allocated.

Dwarf galaxies very close to us such as Sagittarius are also discarded, because owing to the very strong interaction with the MW they are likely to lose their identity very soon crushed by the gravitational interaction.

Finally, we need to avoid the two Magellanic Clouds. Simultaneously dealing with the interaction between the triplet made by the MW and two Magellanic Clouds and the satellite $\mathrm{dG}$ is beyond the technical capability of our numerical code.

The ideal case would be a dG born as a dIrr that after several passages at the peri-galactic changes its morphology and now appears as a dSph still keeping its identity as a dG.

Among the dGs listed in Tables 1 and 2, Sculptor is the best candidate for our experiment. For this galaxy we know the present-day galacto-centric velocity and position of the barycentre. Assuming for simplicity that its orbit has not changed in the past because of dynamical interactions of any kind, we want to prove that on a time scale of a few passages at the peri-center an initial dIrr turned into the Sculptor galaxy we see today. Since there was no change in the orbit, the presentday values of velocity and position can be used to determine the orbit at any time (a crude approximation but fully acceptable in the context of our experiment).

The galaxies of Table 2 were likely generated by objects encompassing a large range of spatial orientations of the main body (represented by the unit vector perpendicular to the plane of symmetry) with respect to the velocity vector of their barycentre. Limited to the case of Draco, Ursa Minor and Sculptor, mass, velocity, and initial position suggest that the future morphological evolution does not much depend on the initial inclination of the initial body (disk) with respect to direction of motion. Most likely, this does not apply to other dGs of the LG (see Mayer et al. 2001a for more details).

\subsection{Orbit of Sculptor}

First, we calculate the orbital motion of the barycentre of Sculptor over a period of about $8 \mathrm{Gyr}$ after $T_{0}$. The time step we have adopted is 0.1 Gyr. Projections of the orbit onto the $X-Y$ and $X-Z$ planes are shown in Fig. 12, whereas the temporal variation of the distance from the Sculptor to the MW is displayed in Fig. 13. The results presented in Sect. 6 below will show that the disk galaxy is heavily distorted by the interaction with the MW and that a large fraction of the mass is lost in long tails and debris, and only a small blob of matter survives gravitationally bound which could be interpreted as the descendent $\mathrm{dSph}$. The orbital motion we are describing refers only to this object.

The orbital motion is confined between $112 \mathrm{kpc}$ (apocenter) and $67 \mathrm{kpc}$ (per-center) with a mean distance to the MW of about $90 \mathrm{kpc}$. It is soon evident that over a time interval of about $8 \mathrm{Gyr}$ (after $T_{0}$ ) the satellite galaxy passes four times at the galactic peri-center with a periodicity of about 2 Gyr. As shown there is no significant variation (decrease) of the orbit peri-center with respect to the MW. This clearly shows that the progenitor of the $\mathrm{dSph}$ maintains a gravitationally bounded core orbiting in the potential well of the MW without suffering effects of dynamical friction which would induce a spiraling motion toward the MW. This confirms that the approach we are using is correct and in agreement with the linear response theory of Colpi (1998) and the assumptions made by Mayer et al. (2001a) in simulations of the same type.

\section{Metamorphosis}

As far as the morphology of the satellite is concerned, two passages at the peri-center are sufficient to make evident the effect we are looking for. The situation is shown in Fig. 14 in which a 3-D large scale view of the satellite orbiting about the MW is given at the age $T_{0}+4 \mathrm{Gyr}$, i.e. after two close passages, and Fig. 15, in which a expantion of the sphere displayed in the Fig. 14 is shown. In Fig. 15 the origin of the reference frame is translated onto the barycentre of the main body of $\mathrm{dG}$. The new frame of reference will be used to analyse the properties of this part of the satellite galaxy (see below).

Examining Figs. 14 and 15 we can compare the structure and evolution of the interacting $\mathrm{dG}$ with that of the isolated case. We can note:

(i) The original dIrr (disk) galaxy has been almost completely torn apart by the gravitational interaction with the MW. This is shown by the long tails of gas, stars, and DM along the orbit of the $\mathrm{dG}$. 

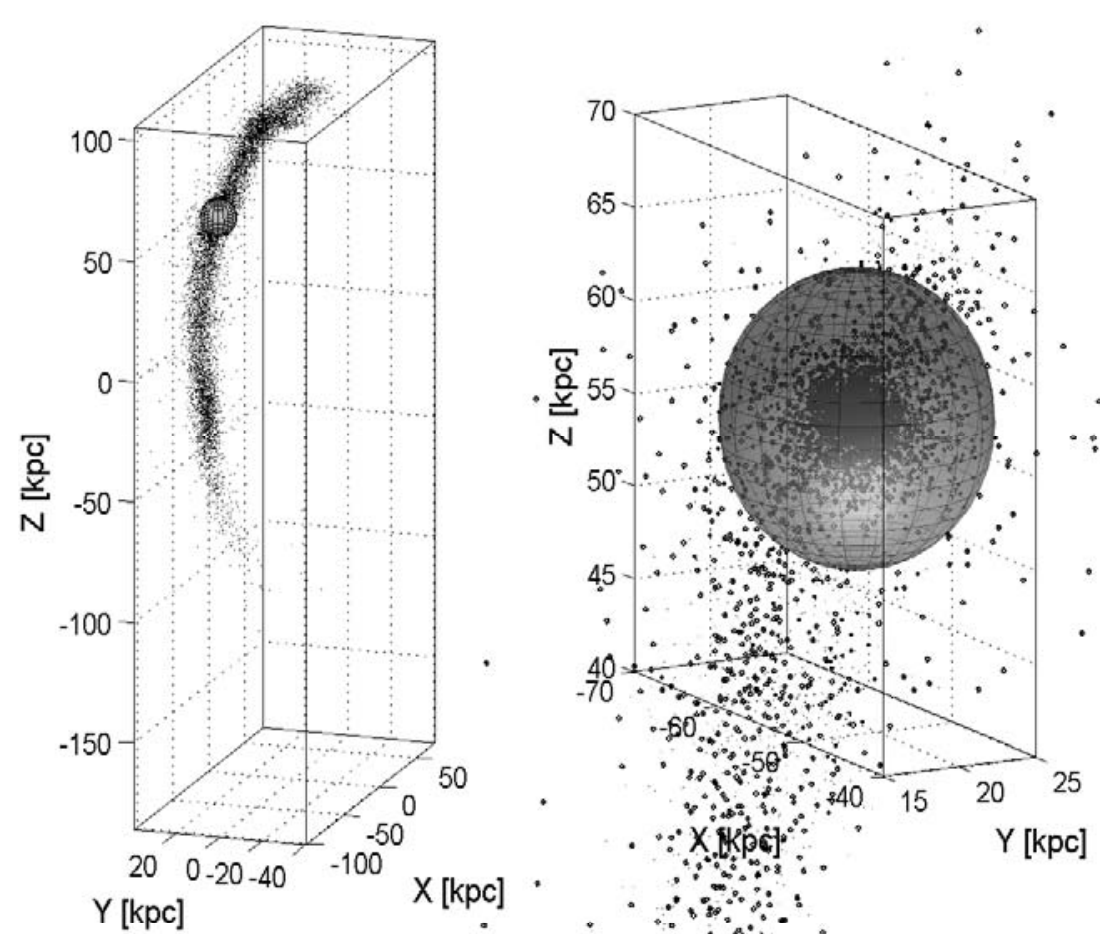

Fig. 14. Left panel: 3-D sketch of the tidal tails of gas, stars, and DM that follow and precede the orbit of the central body of the dG at the age of $T_{0}+4$ Gyr. It shows the position of the dG with respect to the MW locate in the center of the frame of reference. The coordinates of the point of the orthographic projection are $71.5^{\circ}$ azimuth and $14^{\circ}$ elevation. Right panel: expansion of the central body of the dG. Dots are DM, small circle for gas and small cross for star. The coordinates of the point of view of the orthographic projection are $61.5^{\circ}$ azimuth and $12^{\circ}$ elevation. The light grey zone in the sphere shows the position of the foreground MW.

(ii) Nevertheless, a bound blob of gas, stars and DM with total mass of about $1.31 \times 10^{8} M_{\odot}$ and almost round shape survives. Its scale height along the $Z$-axis has indeed increased to about $5 \mathrm{kpc}$, i.e. comparable with its extension along the $X$ and $Y$ directions which has remained nearly unchanged from $T_{0}$ up to $T_{0}+4 \mathrm{Gyr}$. This is clearly shown in the blow-out of Fig. 15. The blob of matter that survived tidal disruption of the original disk galaxy much resembles a dSph. In order to better understand the morphology of the new DSph, in Fig. 15 we present three projections of iso-number density regions in the space of positions limited to the sole stellar component so that direct comparison with isophotal contours is possible. The vertical bar in Fig. 15 indicates the color code for different values of the number density in units of number of stars $\mathrm{kpc}^{-2}$. The projections are for three different angles as indicated. In each projection there is a distinct central concentration of stars with nearly spherical shape surrounded by regions of much lower number density. The projection at $25^{\circ}$ (bottom left) and $45^{\circ}$ (top right) clearly show traces of the tidal tail of stars that is evident in Fig. 14, whereas these are missing in the projection at $80^{\circ}$ (bottom right) where the shape is spherical. The newly born object is indeed a spheroidal galaxy.

(iii) We can compare the mass profile of the new dSph shown in Fig. 16 with the correspond one of Fig. 6. The dSph is still DM dominated but for the very central regions where the baryonic mass prevails; it is almost void of gas (or very little) which has partly converted to stars and partly lost in the wind and tails stripped by the MW. These properties are typical of $\mathrm{dSph}$ and strongly suggest that the kind of metamorphosis indicated by the simulations is at work. The total mass of gas, stars, and DM of the dSph are summarized in Table 4.

(iv) The major change occurring in the $\mathrm{dSph}$ concerns its kinematics, i.e. the profiles of the rotational velocities shown in Fig. 17 for the three components: gas, stars and DM. Compared to the initial ones of Fig. 2, all the three are lower by a factor of 3 indicating that the initial circular motions have been turned into motions equally shared along the three directions. This point will be examined in more detail below. They new values also agree with maximal estimate of circular velocities in dGs by Pedraz et al. (2002).

(v) Finally, we look at the stellar surface brightness profile shown in Fig. 18. Compared to the profile of the initial model and that of the case evolved in isolation (see. Fig. 8), the surface brightness profile of the new dSph tends to be steeper toward the center as often observationally indicated for the $\mathrm{dGs}$ in the Local Group (Mateo 1998). The profile is also compatible with recent determinations of the surface brightness in dEs by Jerjen et al. (2001). 

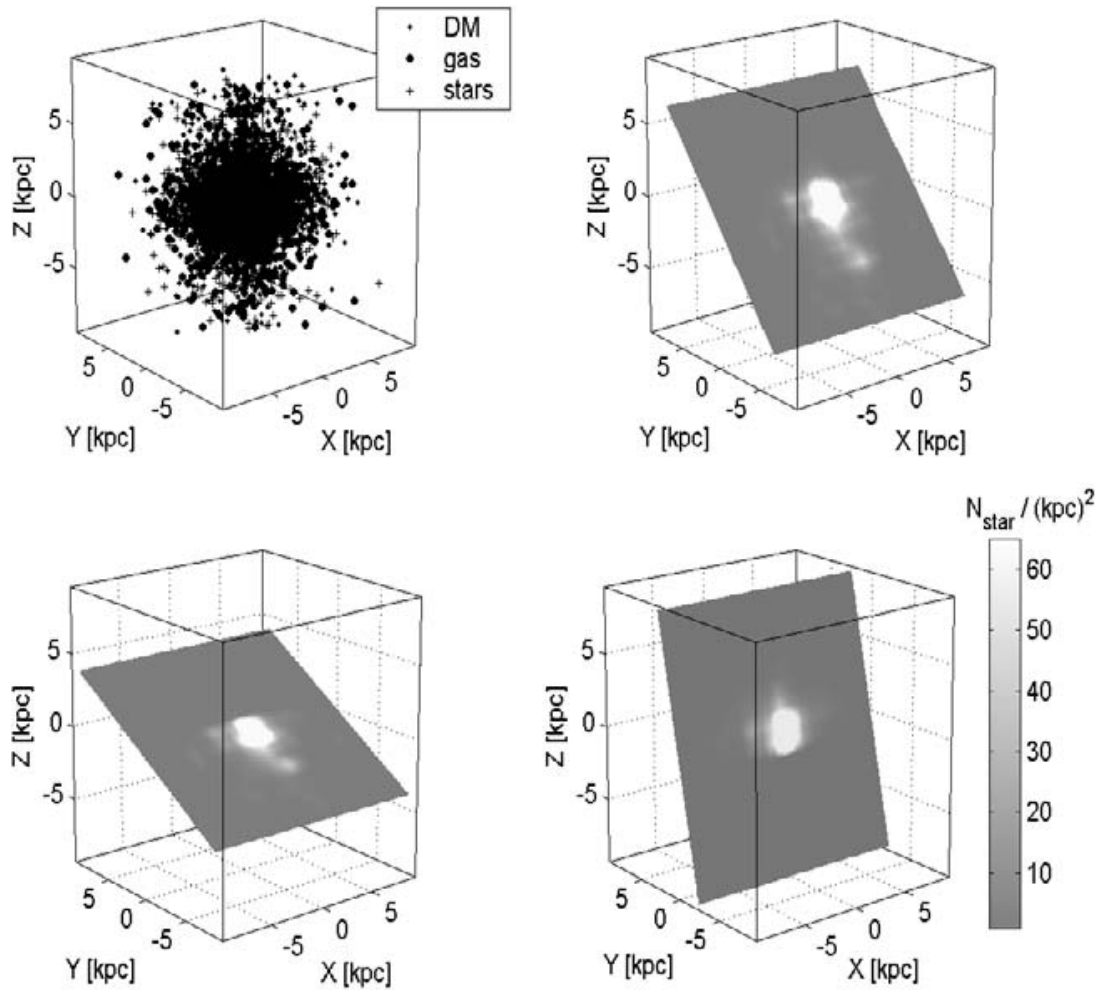

Fig. 15. 3D-view of the central region of the satellite galaxy after 4 Gyr of evolution. The top left panel shows the the central body of the galaxy enclosed in the sphere of Fig. 14, the tidal tail has been removed. the remaining three panels show projections on the planes with different inclinations, i.e., $25^{\circ}$ (bottom left), $25^{\circ}$ (top right) and $80^{\circ}$ (bottom right), the iso-number density of stars in the space of positions. The vertical bar displays the color code corresponding to different values of the number density of stars. The three projections clearly show how the structure of this central region has become nearly spherical. Traces of the tidal tail are visible in the $25^{\circ}$ and $45^{\circ}$ inclination projections. The original disk galaxy has generated a DSph.

(vi) To conclude our quick analysis of the properties of the new dSph we note the short time scale (compared to the Hubble time) required to get the morphological change from a disk galaxy to $\mathrm{dSph}$ resembling object. For the case under consideration the time scale was of the order of two passages past the MW (approximately $4 \mathrm{Gyr}$ or shorter). This secures a posteriori that neglecting dynamical friction, and consequent evolution of the relative distance between the satellite and the MW, and finally the deformation of the MW and consequent spiraling in of the $\mathrm{dG}$ towards this, was not too a bad approximation: see Colpi \& Pallavicini (1998), and Colpi (1998) for more details and a comparison with $N$-body simulations.

(vii) What about the tails of gas stars and DM lost by the parent galaxy and left in orbit around the MW? This material can be captured by the MW itself, or by other dGs. In any case it is no longer considered part of the parent galaxy and as far as the scope of this study are concerned it is left aside. As matter of fact, nothing can be conjectured about its evolution, but see Mayer et al. (2002a) for possible alternatives.

\section{Stability of the new dSph}

An important question to address is whether the structure of the dSph we have obtained after two passages past the MW is

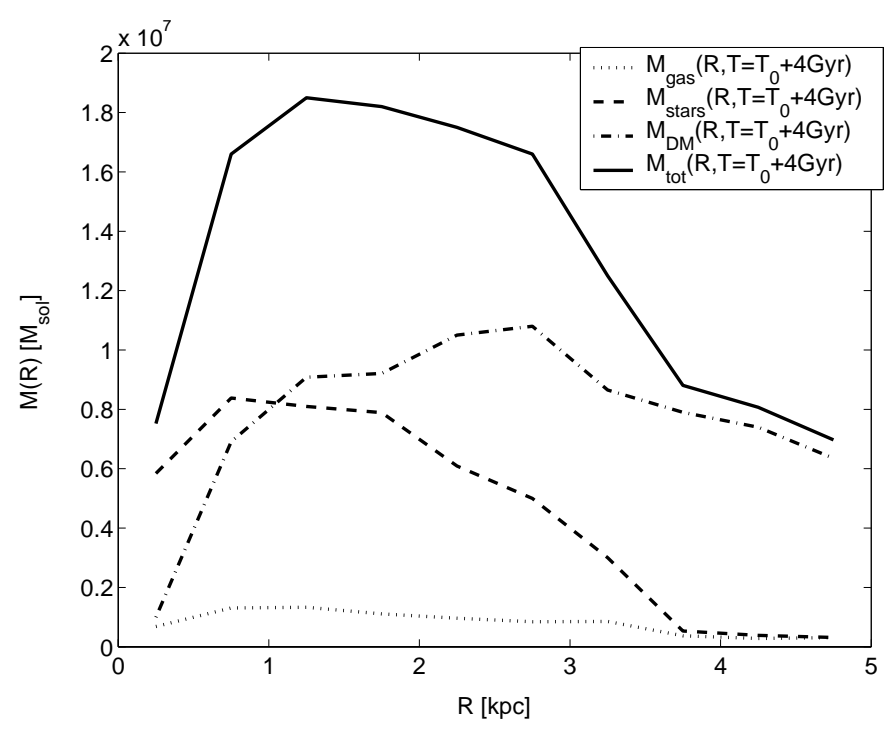

Fig. 16. Radial profiles of the differential distribution of mass (mass per $\Delta R$ bin) in the main body of the satellite galaxy orbiting the MW at the age of $T_{0}+4 \mathrm{Gyr}$. The center of the coordinate system coincides with the barycentre of the central region of the galaxy. The dotted, dashed, dotted-dashed, and solid lines correspond to the gas, stars, DM, and total, respectively.

stable or not. In other words to check whether it survives further evolution and passages. 
Table 4. Properties of the central body of the $\mathrm{dG}$ at the age $T_{0}+4 \mathrm{Gyr}$. The mass resolution is the same as for the data of Table 3. Masses are in $M_{\odot}$, distances and dimensions are in kpc. $M_{\mathrm{s}, \mathrm{T}}$ is the total mass of stars of the dG including $M_{\mathrm{SFR}, \mathrm{T}}$ : the new star formed mass.

\begin{tabular}{ccccc|ccc|ccc}
\hline \hline \multicolumn{4}{c}{ Masses } & \multicolumn{3}{c|}{ Barycentre } & \multicolumn{3}{c}{ Dimensions } \\
\hline$M_{\mathrm{T}}$ & $M_{\mathrm{g}, \mathrm{T}}$ & $M_{\mathrm{s}, \mathrm{T}}$ & $M_{\mathrm{d}, \mathrm{T}}$ & $M_{\mathrm{SFR}, \mathrm{T}}$ & $X_{B}$ & $Y_{B}$ & $Z_{B}$ & $\Delta X$ & $\Delta Y$ & $\Delta Z$ \\
\hline $1.31 \times 10^{8}$ & $8.06 \times 10^{6}$ & $4.55 \times 10^{7}$ & $7.78 \times 10^{7}$ & $1.39 \times 10^{7}$ & -49.4 & 18.3 & 57.1 & 5.0 & 5.0 & 5.0 \\
\hline
\end{tabular}

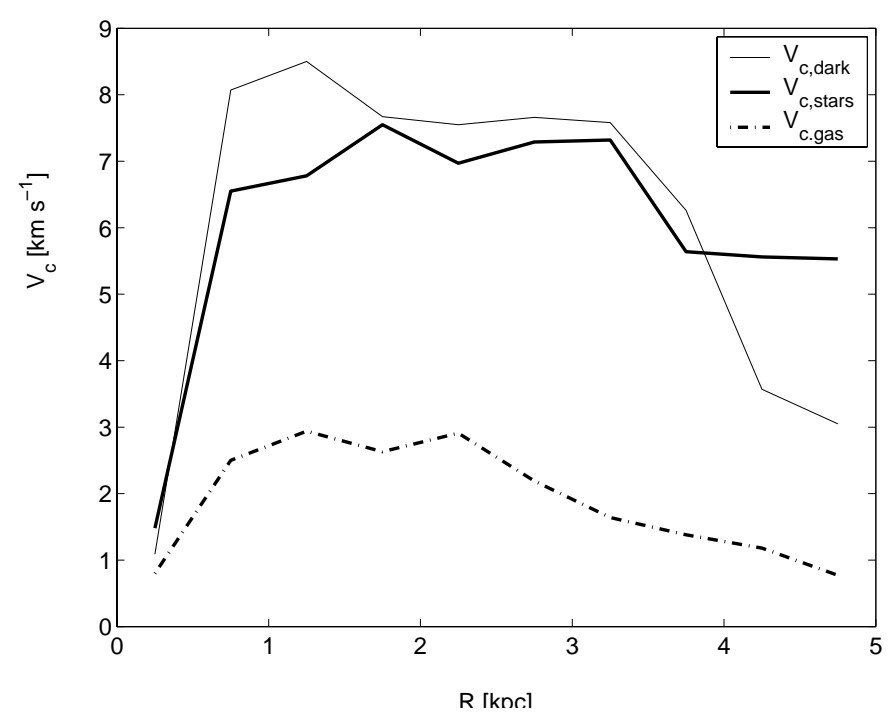

Fig. 17. The radial profiles of circular velocities for gas (dotted-dashed line), stars (heavy solid line) and DM (thin solid line) in the $T_{0}+4 \mathrm{Gyr}$ model of the main body of the $\mathrm{dG}$ shown in shown Fig. 15. The origin of the coordinate system is the same is the same as in Figs. 15 and 16.

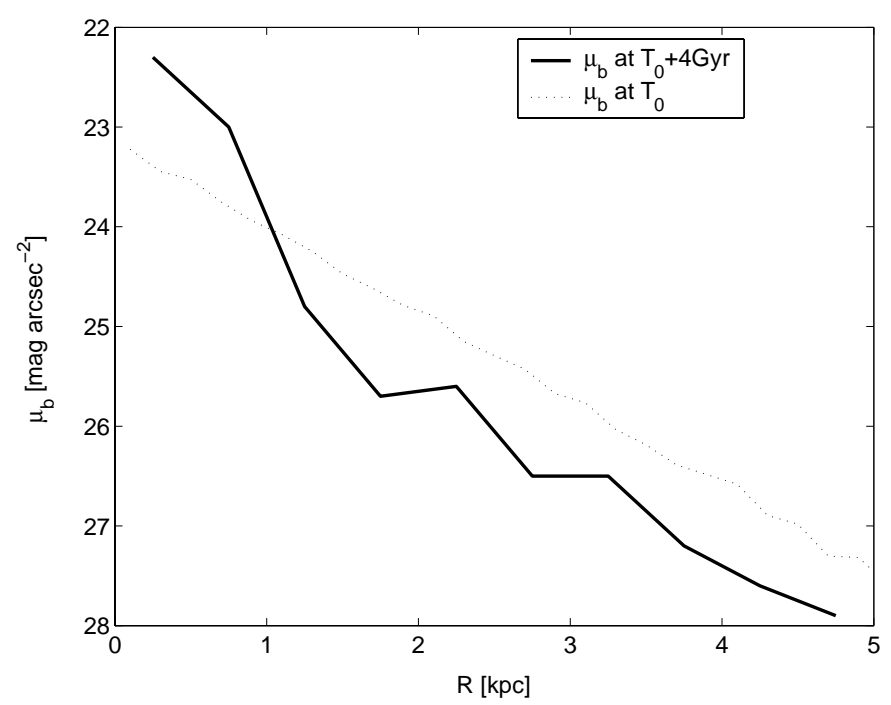

Fig. 18. Surface brightness profile of the model galaxy at the age $T_{0}+$ 4 Gyr confronted with the initial one.

Keeping in mind that a time scale of about $6-8$ Gyr is fully compatible with the time orbital scales of dGs of the Local Group around the MW, we decide to follow the numerical simulations including a third passage past the MW, i.e. up to the age of $T_{0}+6 \mathrm{Gyr}$ and to compare the new model at this age with that at the age $T_{0}+4 \mathrm{Gyr}$.
If few changes happen to occur at the age of $T_{0}+6 \mathrm{Gyr}$, we are confident that what we have obtained at the age of $T_{0}+4 \mathrm{Gyr}$ is a galaxy which has already undergone the morphological transformation and not an object in the course of undergoing it. The results of this experiment are shown in Fig. 19 which is the analog of Fig. 15, whereas a few relevant quantities of this model are presented in Table 5, the analog of Table 4. The blob of matter representing the $\mathrm{dSph}$ is still there, it dimensions are similar to the previous ones, however the total mass, the gas mass and the star mass have been decreased by a factor of about three because further tidal stripping of matter has occurred (see the entries of Table 4). The morphological transformation survives the third passage. We may reasonably conclude that the new dSph is a stable object. The analogs of Figs. 16-18 can be easily obtained with no significant difference but the re-scaling of the masses.

It is worth looking at the evolution of the internal kinematics as a function of time over the $6 \mathrm{Gyr}$ of interest. This is shown in the three panels of Fig. 20, which displays the velocity dispersions along the axes $X, Y$, and $Z, \sigma_{X}$ (top left), $\sigma_{Y}$ (top right), and $\sigma_{Z}$ (bottom), respectively. The velocity dispersions are shown for gas, stars, and DM as indicated. The mean dispersion is nearly the same along the three directions and remains nearly constant with time. The velocity dispersion of DM shows, however, an oscillatory behaviour which is probably caused by the first two passages near the MW. Of particular interest is the slow increase of $\sigma_{Z}$ for the stars and gas, strictly clearly corresponding to the morphological change from a disk to a round object and indicates the gradual isotropization of the structure.

To confirm what we have deduced from the velocity dispersions of Fig. 20, we may also look at the evolution of the total angular momentum. This is shown in Fig. 21. It is soon evident that the decrease in the total angular momentum is very fast during the first 4 Gyr when the dSph is built up, whereas it is slow during the subsequent evolution.

\section{Star formation history of the dwarf galaxy}

In recent years, much effort has been spent to infer the star formation history (SFH) of dGs of the LG from their observed Color-Magnitude diagrams (CMD), owing to the paramount importance of this information to understand the mechanism of galaxy formation, the dGs in particular that are believed to be the building blocks of bigger galaxies. To this purpose, different sophisticated techniques of population synthesis diagnostics have been developed (Gallart et al. 1999; Hernandez et al. 2000, and references therein).

The result of all this is that in many cases the SFH deduced from the CMDs is very irregular, varying from galaxy 
Table 5. Summary of the main properties of the central body of the dwarf satellite at the age $T_{0}+6$ Gyr. The mass resolution is the same as for the data displayed in Table 3. Masses are in units of $10^{6} M_{\odot}$, distances and dimensions are in kpc. $M_{\mathrm{s}, \mathrm{T}}$ is the total mass of stars of the dG inclusive of the mass $M_{\mathrm{NFS}}$ of newly formed stars.

\begin{tabular}{ccccc|ccc|ccc|c|c}
\hline \hline \multicolumn{4}{c|}{ Masses } & \multicolumn{3}{c|}{ Barycentre } & \multicolumn{3}{c|}{ Dimensions } & $\mu_{B}$ & $V_{\mathrm{c}}$ \\
\hline$M_{\mathrm{T}}$ & $M_{\mathrm{g}, \mathrm{T}}$ & $M_{\mathrm{s}, \mathrm{T}}$ & $M_{\mathrm{d}, \mathrm{T}}$ & $M_{\mathrm{NFS}}$ & $X_{B}$ & $Y_{B}$ & $Z_{B}$ & $\Delta X$ & $\Delta Y$ & $\Delta Z$ & $\mathrm{mag} / \mathrm{as}^{2}$ & $\mathrm{~km} \mathrm{~s}^{-1}$ \\
\hline 46.3 & 2.96 & 18.3 & 25.0 & 2.2 & -49.4 & 18.3 & 57.1 & 4.0 & 3.8 & 3.9 & $\geq 22$ & $\leq 4$ \\
\hline
\end{tabular}
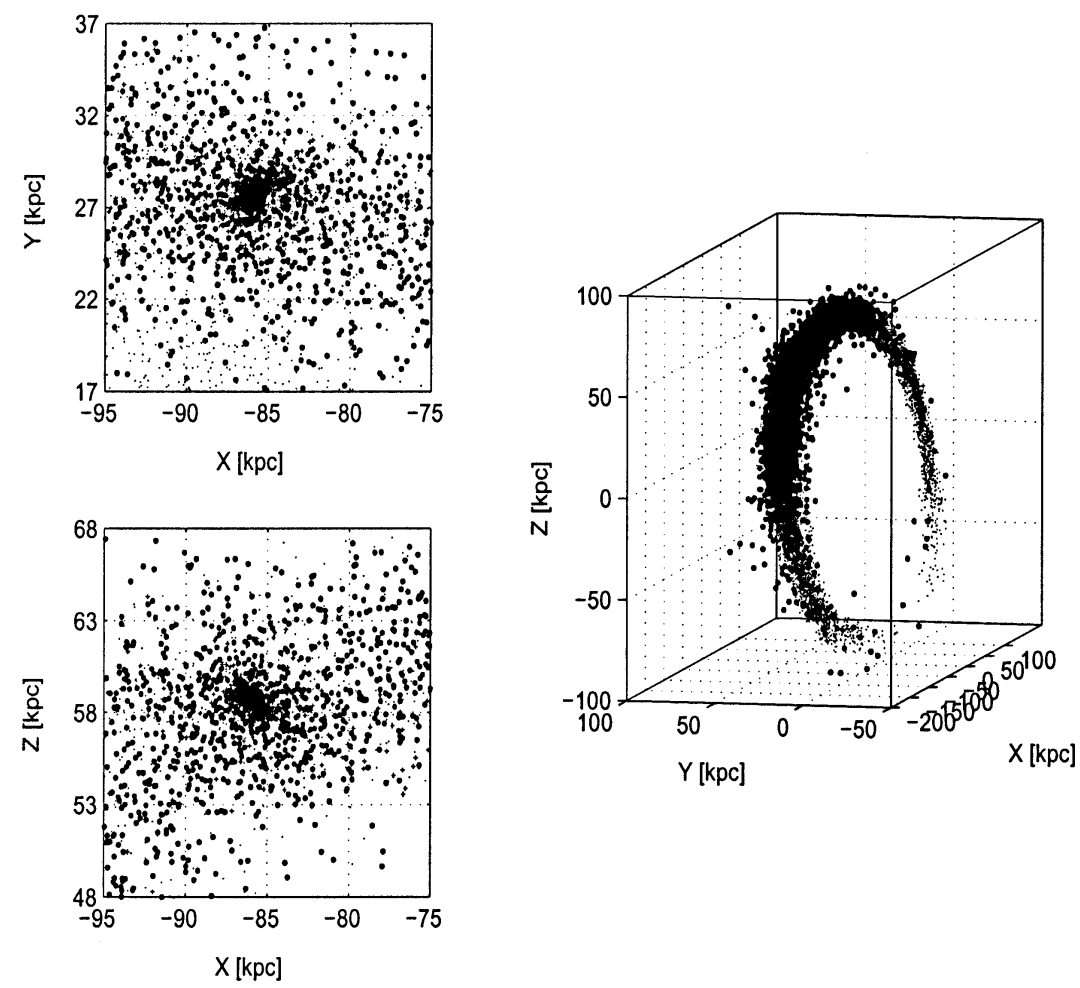

[kpc]

Fig. 19. Structure of the main body of the satellite galaxy orbiting the MW at the age of $T_{0}+6$ Gyr. Left panels: projections onto the $X Y$ (top) and $X Z$ (bottom) planes. Right panel: 3D orthographic projection as seen from $-69^{\circ}$ azimuth and $14^{\circ}$ elevation of the satellite and its tails. In each panel, stars, gas and DM particles are indicated with crosses, circles, points, respectively.

to galaxy. Nice examples are the dSph galaxies Leo I (Gallart et al. 1999), Carina and Sculptor (Smecker-Hane \& McWilliam 1999): Leo I in addition to a very old population shows the signature of recent star forming activity; in Carina, star formation seems to have lasted more than about $6 \mathrm{Gyr}$; in the case of Sculptor a dominant old burst of relatively short duration seems to be more appropriate. Initial, short-lived star forming activity is also typical of other galaxies like Draco and Ursa Minor whose CMDs resemble those of globular clusters in the MW Halo.

Is the SFH related to intrinsic properties of $\mathrm{dGs}$ or is it the result of dynamical interactions with other galaxies of the LG? This possibility has indeed been put forward (see Mateo 1998) arguing that dGs were born almost at the same time, but evolved along different paths, probably depending on their distance to the MW, their orbit and the amount of interaction experienced in the past. Is this the sole possibility?

Carraro et al. (2001) and Chiosi \& Carraro (2002) have addressed the whole subject looking for an explanation of the large variety of SFH in galaxies of different mass (luminosity) based on first principles. Assuming early virialization of DM haloes and using the $N$-body Tree-SPH technique they found that the SFH of galaxies obeying the so-called monolithic scheme (no hierarchical aggregation of smaller bodies into a big galaxy) gradually changes from a single sharply peaked initial episode to a series of bursts stretching over most of the Hubble time at decreasing galaxy mass and/or initial density of the proto-galaxy. In the case of dGs, simulations of their CMDs have shown that agreement is possible, e.g. the CMD of Sculptor was reproduced by a model galaxy with relatively low initial density (Carraro et al. 2001).

The question to be addressed here is whether the dynamical interactions with a big galaxy (the MW in our case) may stimulate further stellar activity in the satellite in addition to the spontaneous one. The expectation is that at each close encounter a relative peak in rate of star formation should occur. Therefore dGs should undergo fluctuating stellar activity of both internal and external origin. The internal one is caused by the interplay of several concurring and contrasting effects such as the energy feed-back by supernova explosions, chemical 

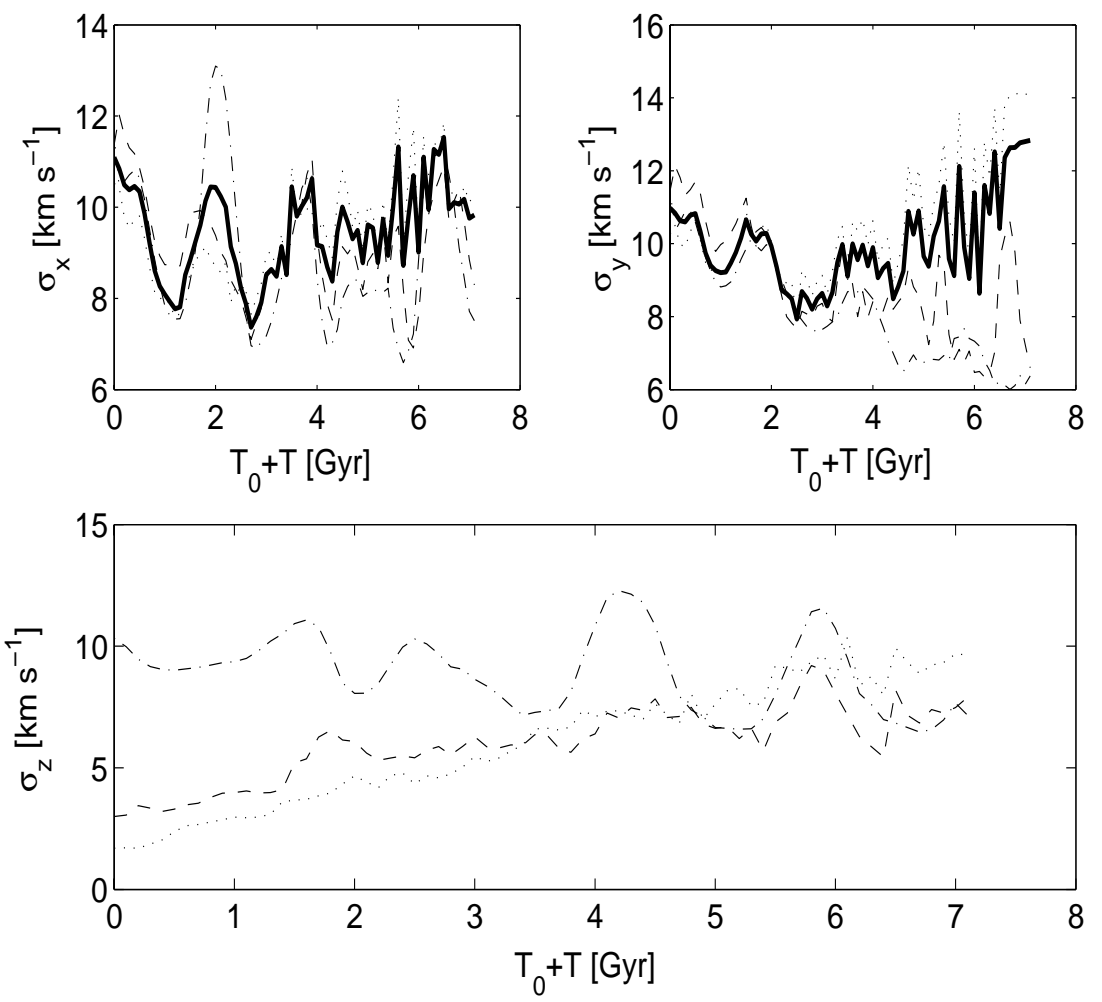

Fig. 20. Evolution of the velocity dispersions. The upper left panel shows the dispersion along $X$ direction, the upper right is the same but for the $Y$ direction. The lower panel shows the dispersion along the $Z$ direction, showing the steady increase of the mean $\sigma_{Z}$ of gas and stars induced by the tidal interaction with the MW. The meaning of the symbols in the various panels is as follows: the thick line is the total velocity dispersion, the dotted dashed line is for DM, the dotted line is for stars, the dashed line is for gas, and the dotted-dashed line is for DM.

enrichment, cooling, total gravitational potential, and finally the galactic wind. The rules of this game are described in detail by Chiosi \& Carraro (2002) to whom the reader should refer. The external one, stimulated by the stronger interaction at the close encounters, should occur via perturbations of the gas density.

Before proceeding further, it is worth commenting here on a few technical details that are needed to correctly evaluate the SFR in a galaxy that changes mass, dimensions, and morphology. In the course of its life, the galaxy can both acquire and lose stars. Thanks to the approximation adopted for the MW, i.e. a vectorial field of force, all stars in the simulation belong to the satellite. They have been formed both in the early epochs $\left(T \leq T_{0}\right)$ and while orbiting around and interacting with the MW.

Since there is no acquisition either from the MW or the ambient medium, but only loss of stars in tails and debris, one has to consider only the stars that are currently contained in the dSph. From a technical point of view the SFR can be correctly evaluated in the following way: we start from the number of gas and star particles in the satellite which, as shown in Fig. 22, are expected to decrease because of the various losses of material and normalize them to their sum (also decreasing) to obtain the fractionary mass of stars contained in the satellite (the removal of those lost in the tails is implicit).

With this procedure we immediately obtain the SFR relative to the sole dSph and the total mass of its stellar content. Out of the $4.55 \times 10^{7} M_{\odot}$ in the final total mass of dSph at $T_{0}+4 \mathrm{Gyr}$,

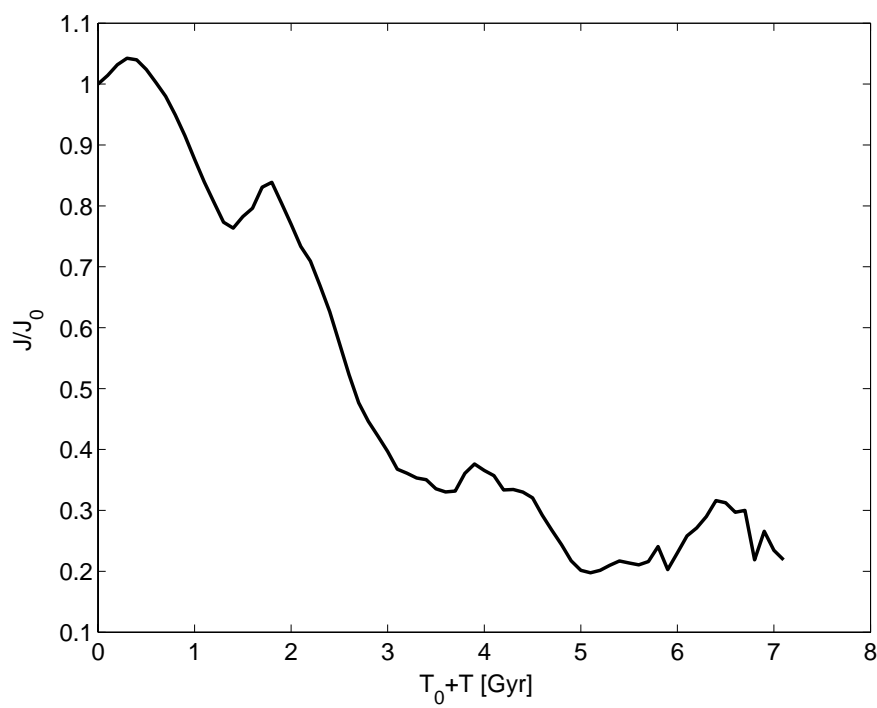

Fig. 21. The temporal evolution of the total angular momentum $J$ of the $\mathrm{dG}$ normalized to the initial value $J_{0}$. The fast decrease is followed by less steep variation once the round central body has been generated, starting from an age of about $T_{0}+3.5 \mathrm{Gyr}$.

$1.39 \times 10^{7} M_{\odot}$ are of new born stars. Similarly in the galaxy evolved up to $T_{0}+6 \mathrm{Gyr}$ the mass of new borne stars amounts to $2.1 \times 10^{6} M_{\odot}$.

The rate of SF of the satellite galaxy as a function of time during the interval $T_{0}$ to $T_{0}+6 \mathrm{Gyr}$ is shown Fig. 23 (bottom panel) together with the SFR history for the galaxy evolved in 

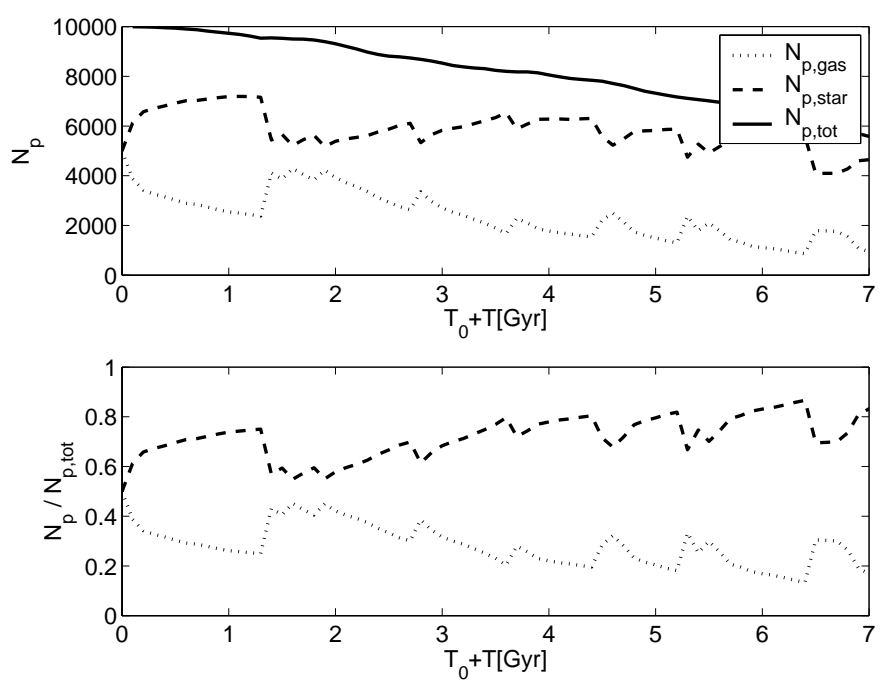

Fig. 22. Top panel: variation of the current number of particles with respect to the total number of particles in the simulation. The dotted, dashed and solid lines are for the gas, stars and total, respectively. Bottom panel: the same but normalized to the total number of particles locked up in the main body of the newly borne dSph.

isolation (upper panel). The initial activity (the one building up the dIrr-disk galaxy) is followed by several bursts of variable intensity which seem to take place randomly in time. The major difference between the two cases is that for ages older than about $1 \mathrm{Gyr}$, while the isolated galaxy undergoes only minute star formation, the one interacting with the MW has more intense stellar activity. On the average, the intensity is comparable to the mean value during the initial period (ages younger than about $1 \mathrm{Gyr}$ ). It seems therefore that the intensity of star formation is somehow sensitive to the interaction. However, as far as the correlation (if any) of the star forming bursts with the orbital position of the satellite with respect to the MW (cf. Mayer et al. 2001b) it seems that the most intense ones are favored when the satellite galaxy is at the peri-galactic. The preliminary conclusion would be that SF is mainly driven by internal processes. It might be that the failure of tidal interactions in triggering strong bursts of SF is due to the fact that the encounters are still too far away so that the gravitational interaction is sufficient to alter the dynamical structure of the $\mathrm{dG}$ but not to induce gas density perturbations strong enough to increse the rate of SF. Alternatively, the passage to the pericenter of the orbit increases the kinematical temperature of the gas which becomes less dense so that star formation is less efficient. Furthermore, it might be that the rate of star formation we have adopted is missing an important term related to the tidal interaction. In this context it worth recalling that the Schmidt law not only is a crude approximation of the complexity of the star forming processes but also neglects all possible effects due to the environment, in particular those of cosmological nature, see for instance Gnedin (2000) for a discussion of this topic. Finally, one should not forget that some uncertainty in the derivation of the SFR from $N$-body simulation is always present as pointed out by Hirashita (1999).

Another obvious criticism to the above picture would be that we are using disk galaxies instead of genuine dIrrs and that
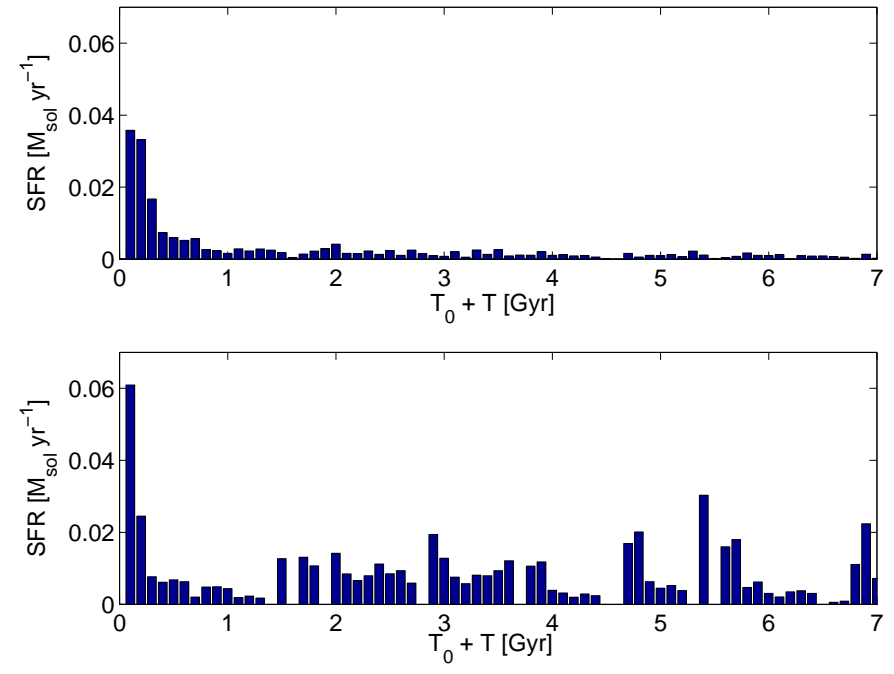

Fig. 23. Star Formation Rate of the $\mathrm{dG}$ as function of time. The upper panel shows the refers to the galaxy evolved in isolation. The lower panel displays the same but for the $\mathrm{dG}$ orbiting around and interacting with the MW. After the initial activity, a number of small amplitude bursts of star formation seem to occur. See the text for more details.

perhaps with these latter more important effects on the SFH are possible. As far as the stellar content of Sculptor is concerned, model galaxies like those in Carraro et al. (2001) are perhaps better suited to match the present day CMD. Model B1 of Carraro et al. (2001) characterized by the initial $M_{\mathrm{G}}=10^{9} M_{\odot}$, $M_{\mathrm{DM}}=0.9 \times 10^{9} M_{\odot}, M_{\mathrm{g}}=0.1 \times 10^{9} M_{\odot}$, low mean density, and an initial episode of star formation lasting about $2 \mathrm{Gyr}$, if captured by the MW during the initial period of activity (thus resembling a dIrr), losing mass and angular momentum by tidal interaction could correspond to Sculptor. However, even model B1 is not entirely suited to our purposes because it was calculated in absence of rotation. The ideal case would be a rotating, low initial density model, whose complete evolution is followed in detailed by $N$-body Tree-SPH numerical calculations. The subject is left to future investigation.

\section{Discussion and conclusions}

Before drawing any conclusion it is worth commenting on the major points of uncertainty affecting our study:

(i) The rotation curves of dIrrs suggest that their halos have nearly constant density profiles in the core. Models in the standard Cold Dark Matter scenario are expected to possess the Hernquist mass density profiles which are much steeper that those derived from the rotation curves. In relation this, to see the studies by Firmani et al. (2000) for self-interacting DM models, Bode et al. (2001) warm DM models, and Peebles (2000) for fluid DM models.

(ii) The simulations are not framed in a model of cosmological evolution of galaxies, in which masses, dimensions, gravitational potentials, SF law, are functions of the redshift in order to fully and properly describe the effects of gravitational interactions on the orbit and internal dynamics of the satellite galaxy. For instance the possibility that the gravitational potential exerted by the MW has 
varied with time because of mass accumulation by capture of smaller bodies. Furthermore, re-scaling masses, relative distances and gravitational potential back into the past could also affect the morphological evolution of the $\mathrm{dG}$ we see today. A smaller mass, a shorter relative distance, and a weaker gravitational potential for the MW could indeed lead to a smaller effect on the dynamical structure and morphological evolution of the dG. In any case, according to Mayer et al. (2001a), tidal re-shaping of dGs should always be important.

(iii) No detailed chemical evolution in the galaxy models has been taken into account because we were primarily interested in the morphological effects caused by the dynamical interaction. However, a detailed treatment of chemical enrichment bears very much on the galactic models via the effect on cooling and star formation in turn.

Given these premises, the results we have obtained can be summarized as follows:

(1) The morphological transformation of a bigger disk-like (perhaps dIrr) galaxy into a smaller dSph is possible on a time scale of about 4 Gyr (or even less) thanks to the dynamical interaction with the MW under suitable conditions regulating the orbital motion of the satellite around the host galaxy. This time scale is shorter than suggested by Mayer et al. (2001a) who, however, analyzed the problem from a wider point of view.

(2) The resulting dSph seems to be stable. As a matter of fact, it survives another close encounter at least. Actually this helps the dSph to get a total mass in closer agreement with the observational estimates for dGs of the LG (Mateo 1998).

To conclude, despite the many limitations and uncertainties affecting our analysis, at a reasonable level of confidence the present simulations show that tidal interactions may re-shape a dIrr (disk-like) galaxy into a DSph.

Acknowledgements. We would like to thank the anonymous referee for his/her useful criticism and remarks that much improved the original version of the paper. This study has been financed by the Italian Ministry of Education, University, and Research (MIUR), and the University of Padova under the special contract "Formation and evolution of early type galaxies".

\section{References}

Allen, C., \& Martos, M. A. 1986, RMAA, 13, 137

Allen, C., \& Santillan, A. 1991, RMAA, 22, 255

Athanassoula, E., \& Sellwood, J. A. 1986, MNRAS, 212, 195

Bahcall, J. N., \& Soneira, R. M. 1980, ApJS, 44, 73

Barazza, F. D., Binggeli, B., \& Jerjen, H. 2002, A\&A, 391, 823

Barnes, J., \& Hut, P. 1986, Nature, 324, 446

Bekki, K., \& Chiba, M. 2000, ApJ, 534, L89

Bekki, K., \& Chiba, M. 2001, ApJ, 558, 666

Benz, W. 1990, in Numerical Modelling of Nonlinear Stellar Pulsation, ed. J. R. Buchler (Dordrecht: Kluwer), 269

Bertschinger, E. 2001, MNRAS, 137, 1

Binney, J., \& Merrifield, M. 1998, Galactic Astronomy (Princeton University Press)
Binney, J., \& Tremaine, S. 1994, Galactic Dynamics (Princeton University Press), third printing with corrections

Binney, J. 2000, Star2000 - Dynamics of Star Clusters and Milky Way, ed. R. Spurzem, \& R. Wielen, ASP Conf. Ser., 228, 269

Blitz, L., Spergel, D. N., Teuben, P. J., Hartmann, D., \& Burton, W. B. 1999, ApJ, 514, 818

Bode, P., Ostriker, J., \& Turok, N. 2001, ApJ, 556, 93

Bontekoe, T. R., \& van Albada, T. S. 1987, MNRAS, 224, 349B

Buonomo, F., Carraro, G., Chiosi, C., \& Lia, C. 2000 MNRAS, 312, 371

Carollo, C. M., de Zeeuw, P. T., \& van der Marel, R. P. 1995, MNRAS, 276,1131

Carraro, G., Chiosi, C., Girardi, L., \& Lia, C. 2001, MNRAS, 327, 69

Carraro, G., Lia, C., \& Chiosi, C. 1998, MNRAS, 297, 1021

Chandraseckhar, S. 1941, ApJ, 93, 285

Chandraseckhar, S. 1941, ApJ, 93, 30

Chandraseckhar, S. 1941, ApJ, 93, 323

Chandraseckhar, S. 1941, ApJ, 94, 511

Chandraseckhar, S. 1943, ApJ, 97, 255

Chiosi, C., Bressan, A., Portinari, L., \& Tantalo, R. 1998, A\&A, 339, 355

Chiosi, C., \& Carraro, G. 2002, MNRAS, 335, 335

Colpi, M., \& Pallavicini, A. 1998, ApJ, 502, 150

Colpi, M. 1998, ApJ, 502, 167

Colpi, M., Mayer, L., \& Governato, F. 1999, ApJ, 525, 720

Cote, S., Freeman, K., \& Carignan, C. 1997, in Dark and Visible Matter in the Universe, ed. M. Persic, \& P. Salucci, ASP Conf. Ser., 117,52

Cora, S. A., Muzzio, J. S., \& Vergne, M. M. 1997, MNRAS, 289, 253

Da Costa, G. S. 1990, The Magellanic Clouds, ed. R. Haynes, \& D. Milne, (Dordrecht: Kluwer Academic Publishers), 183

Dehen, W. 1999, AJ, 118, 1201

Dehnen, W., \& Binney, J. 1998, MNRAS, 294, 429

De Rijcke, S., Dejonghe, H., Zeilinger, W. W., \& Hau, G. K. T. 2001, ApJ, 559, L21

Ferguson, H. C., \& Binggeli, B. 1994, A\&ARv, 6, 67

Freeman, K. C. 1970, ApJ, 160, 811

Firmani, C., D’Onghia, E., Avila-Reese, V., Chincarini, G., \& Hernandez, X. 2000, MNRAS, 315, L29

Gallart C., Freedman, W. L., Aparicio, A., Bertelli, G., \& Chiosi, C. 1999, AJ, 118, 2245

Gingold, R. A., \& Monaghan, J. J. 1977, MNRAS, 181, 375

Gnedin, O. Y., \& Ostriker, J. P. 1999a, ApJ, 513, 629

Gnedin, O. Y., Hernquist, L., \& Ostriker, J. P. 1999b, ApJ, 514, 109

Gnedin, N. Y. 2000, ApJ, 535, L75

Grebel, E. K. 1999, in The Stellar Content of Local Group Galaxies, ASP San Francisco CA, ed. P. Whitelock, \& R. cannon, 17

Green, R. G. 1985, Spherical Astronomy (Cambridge University Press)

Han, C., \& Ryden, B. S. 1994, ApJ, 433, 80

Hernandez, X., Gilmore, G., \& Valls-Gabaud, D. 2000, MNRAS, 317, 831

Hernquist, L. 1990, ApJ, 356, 359

Hernquist, L. 1993a, ApJ, 404, 717

Hernquist, L. 1993b, ApJS, 86, 389

Hernquist, L., \& Weinberg, M. D. 1989, MNRAS, 238, 407

Hirashita, H. 1999, ApJ, 520, 607

Hoffman, G. L., Salpeter, E. E., Farhat, B., et al. 1996, ApJS, 105, 269

Huang, S., \& Carlberg, R. G. 1997, AJ, 480, 503

Jerjen, H., Rekola, R., Takalo, L., Coleman, M., \& Valtonen, M. 2001, A\&A, 380, 90J

Irwin, M. 1998, IAUS, 192, 29

Johnson, D. R. H., \& Soderblom, D. R. 1987, AJ, 93, 864 
Jog, C. J. 1996, MNRAS, 278, 209

Jog, C. J. 2000, BASI, 28, 309

Katz, N. 1992, ApJ, 391, 502

Katz, N., Weinberg, D. H., \& Hernquist, L. 1996, ApJS, 105, 19

Kawata, D. 2001, ApJ, 548, 717

Klypin, A., Kravtsov, A. V., Valenzuela, O., \& Prada, F. 1999, ApJ, 522,82

Knebe, A., Kravtsov, A. V., Gottlober, S., \& Klypin, A. A. 2000, MNRAS, 317, 630

Kormendy, J. 1977, ApJ, 218, 333

Kormendy, J. 1987, Nearly Normal Galaxies, ed. S. M. Faber (Berlin: Springer), 163

Kormendy, J., \& Djorgovski, S. 1989, ARA\&A, 27, 235

Kroupa, P., \& Bastian, U. 1997, New Astron., 2, 77

Lewis, J. R., \& Freeman, K. C. 1989, AJ, 97, 139

Leeuwin, F., \& Combes, F. 1997, MNRAS, 284, 45

Lia, C., Portinari, L., \& Carraro, G. 2002, MNRAS, 330, 821

Lin, D. N. C., \& Tremaine, S. 1983, ApJ, 264, 364J

Lucy, L. 1977, AJ, 82, 1013

Mac Low, M. M., \& Ferrara, A. 1999, ApJ, 513, 142

Marzke, R. O., \& Da Costa, L. N. 1997, AJ, 113, 185

Mateo, M. 1998, ARA\&A, 36, 435

Mayer, L., Governato, F., Colpi, M., et al. 2001a, ApJ, 559, 754

Mayer, L., Governato, F., Colpi, M., et al. 2001b, ApJ, 547, L123

Mayer, L., Moore, B., Quinn T., Governato, F., \& Standel, J. 2002a, MNRAS, 548, 703

Mayer, L., Moore, B., Quinn T., Governato, F., \& Standel, J. 2002b, MNRAS, 336, 119

Mighell, K., \& Bruke, C. J. 1999, AJ, 118, 366

Miyamoto, M., \& Nagai, R. 1975, PASJ, 27, 533

Monaghan, J. J., \& Lattanzio, J. C. 1985, A\&A, 149, 135

Moore, B., Katz, N., \& Lake, G. 1996a, ApJ, 457, 455

Moore, B., Katz, N., Lake, G., Dressler, A., \& Oemler, A. Jr. 1996b, Nature, 379, 613

Moore, B., Lake, G., \& Katz, N. 1998, ApJ, 495, 139

Moore, B., Ghigna, S., Governato, F., et al. 1999b, ApJ, 524, 19

Moore, B., Lake, G., Quinn, T., \& Stadel, J. 1999a, MNRAS, 304, 465

Murali, C. 2000, ApJ, 529, L81

Navarro, J. F., \& White, S. D. M. 1993, MNRAS, 265, 271
Nelson, R. P., \& Papaloizou, J. C. B. 1994, MNRAS, 270, 1

Pedraz, S., Gorgas, J., Cardiel, N., Sánchez-Blázquez, P., \& Guzmán, R. 2002, MNRAS, 332L, 59P

Peebles, P. J. E., Melott, A. L., Holmes, M. R., \& Jiang, L. R. 1989, ApJ, 345, 108

Peebles, P. 2000, ApJ, 534, L127

Pichon, C., \& Cannon, C. R. 1997, MNRAS, 291, 616

Prugniel, Ph., \& Combes, F. 1992, A\&A, 259, 25

Quinn, P. J., \& Goodman, J. 1986, ApJ, 309, 472

Romeo, A. B. 1992, MNRAS, 256, 307

Schmidt, M. 1959, ApJ, 129, 243

Smecker-Hane, T., McWilliam, A. 1999, Spectro-photometric dating of stars and galaxies, ed. I. Hubney, S. R. Heap, \& R. H. Cornett, ASP Conf. Ser., 192, 251

Spitzer, L. Jr. 1941a, ApJ, 93, 369

Spitzer, L. Jr. 1941b, ApJ, 94, 232

Spitzer, L. Jr. 1942, ApJ, 95, 329

Spitzer, L. Jr., \& Hart, M. H. 1971, ApJ, 164, 399

Toomre, A. 1963, ApJ, 138, 385

Toomre, A. 1964, ApJ, 139, 1217

Tremaine, S. D. 1975, ApJ, 203, 72

van den Bergh, S. 1999, A\&ARv, 9, 273

van den Bergh, S. 1996, PASP, 108, 986

van der Kruit, P. C., \& Searle, L. 1981a, A\&A, 95, 105

van der Kruit, P. C., \& Searle, L. 1981b, A\&A, 95, 116

van der Kruit, P. C., \& Searle, L. 1982a, A\&A, 110, 61

van der Kruit, P. C., \& Searle, L. 1982b, A\&A, 110, 79

Villumsen, J. V. 1985, ApJ, 290, 75

White, S. D. M. 1976, MNRAS, 174, 467

White, S. D. M. 1978, MNRAS, 184, 185

White, S. D. M. 1983, ApJ, 274, 53

White, S. D. M., \& Rees, M. J. 1978, ApJ, 183, 341

Weinberg, M. D. 1989, MNRAS, 239, 549

Weinberg, M. D. 1994a, AJ, 108, 1398

Weinberg, M. D. 1994b, AJ, 108, 1403

Weinberg, M. D. 1994c, AJ, 108, 1414

Widrow, L. M. 2000, ApJS, 131,39

Wilkinson, M. I., \& Evans, N. W. 1999, MNRAS, 310, 645 